\title{
Collusion and the Organization of Delegated Expertise*
}

\author{
Denis Gromb $b^{\ddagger}$ and David Martimort ${ }^{\S}$
}

January 11, 2007

\footnotetext{
${ }^{*}$ Correspondence: Denis Gromb, London Business School, Regent's Park, London NW1 4SA, UK. Email: dgromb@london.edu. Ph: +44-20-7262-5050. Fax: +44 -20-7724-3317.

We thank Marie-Laure Allain, Mark Armstrong, Irina Bogatcheva, Juan Carillo, Hendrik Hakenes, Li Hui, Rongkang Kang, Godfrey Keller, Jean-Jacques Laffont, Christian Laux, Jonathan Levin, Gyöngyi Lóránth, George Mailath, Eric Maskin, Lisa Oliver, Marco Ottaviani, Jean Tirole, Dimitri Vayanos, and two anonymous referees, as well as seminar participants at ATOM Paris, Besançon, CERNA Paris, Chicago, ETAPE Paris, ECARES Brussels, Frankfurt, HEC Paris, IDC Herzliya, Institut Henri Poincaré Paris, the LAMES in Sao Paulo, London Business School, London School of Economics, Munich, Naples, Oxford, Stockholm School of Economics, UCL, Wuhan University and the Econometric Society European Meeting 2003 in Stockholm for discussions and comments. We also thank Marie-Pierre Boé and Cathy Wilson for their help in preparing the manuscript. We are particularly grateful to Ben Polak (the associate editor) for tremendously helpful suggestions and comments. All errors are ours.

${ }^{\ddagger}$ London Business School and CEPR.

${ }^{\S}$ Université de Toulouse I (GREMAQ, IDEI), Institut Universitaire de France, and CEPR.
} 


\begin{abstract}
We study the optimal design of incentive contracts for experts in different collusion environments, and explore implications for the organization of delegated expertise. We consider a principal relying on experts to gather and report two signals about a project's value. The principal can have a single expert gather both signals or two experts gather one signal each. We show that absent collusion, the multiexpert organization dominates the single expert organization. However, this ranking is reversed when the experts can collude among themselves (horizontal collusion) and with the principal (vertical collusion).
\end{abstract}

JEL classification: D81; D82; D86; L23

Keywords: Expertise, Organization, Collusion

Running title: Collusion and Delegated Expertise 


\section{Introduction}

With scientific and technological boundaries moving constantly, decision-making has come to rely increasingly on expertise. Decision-makers do not always have the time and skills to gather and process information on complex issues. Instead, this task is often delegated to experts.

How can one ensure that experts provide accurate information? How accountable should experts be for their recommendations? How should the delegation of expertise be organized? Should one rely on one or several experts? Should the experts' reports be public or private? To address these issues, one must realize that delegated expertise involves an incentive problem between principals and experts, with specific features. Indeed, because experts produce a non-verifiable output, namely information, they must be given incentives not only to acquire information, but also to report it truthfully. An efficient design of incentives for experts must therefore solve an intricate problem mixing both moral hazard and adverse selection.

We study the optimal design of incentive contracts for experts and the organization of delegated expertise in different collusion environments. We consider a principal relying on experts to gather and report two signals about a project's value. The principal can have a single expert gather both signals or two experts gather one signal each. We focus on how the experts' ability to collude among themselves (horizontal collusion) and/or with the principal (vertical collusion) affects the relative performance of these two organization forms. We show that absent collusion, the two-expert organization dominates the one-expert organization, but that this ranking is reversed if both horizontal and vertical collusion are simultaneously possible.

The model is as follows. A risk-neutral principal has a project with two possible outcomes: success or failure. To decide whether to undertake it, he gains access to two noisy signals. The signals are independent conditional on the project's outcome and otherwise identical. Each signal can take two values, positive or negative, and the project is valuable only if both signals are positive. For simplicity, both signals have to be gathered simultaneously.

The principal does not have direct access to the signals. Instead, he must hire one or two risk-neutral experts to gather and report the signals. This task being costly, the experts must be motivated with incentive contracts. The incentive problem is compounded by the fact that reports are fully manipulable, i.e., they need not coincide with the signals observed, and that the experts have limited liability. This allows the experts to derive rents. We study and compare the rents under the two organization forms, i.e., one vs. two experts.

We first study the one-expert/two-signals case. A priori, contracts specify transfers contingent on both reports (each being positive or negative) and the project's outcome (success, failure or rejection). However, because reports are manipulable, the contract cannot distinguish between the expert having observed two negative signals and his having observed one positive and one negative signal. The principal can only use outcome-based contracts. We identify a key 
property of delegated expertise: diseconomies of scale due to agency costs. That is, the expert's rent with two signals is more than twice his rent with just one signal. Indeed, because he is rewarded when his report pair is confirmed by the outcome, an expert is reluctant to gather a second signal because it might conflict with the first one. Motivating the expert to gather more information is increasingly costly for the principal. To reduce agency costs, the principal may forego the benefit of more information and make less than optimally informed decisions.

We then consider the two-experts/two-signals case assuming no collusion. An expert's contract can specify transfers contingent not only on his own report but also on the other expert's, and on the outcome. Such report-based contracts can exploit the signals' correlation through cross-checking: The optimal contract is for each expert to be rewarded only if his report is confirmed by the outcome and if it coincides with the other expert's. This yields economies of scale due to agency costs, which implies that absent collusion, the optimal organization of delegated expertise is for the principal to rely on two experts rather than one.

The performance of the two-expert organization is however sensitive to the collusion environment. Indeed, contracts rewarding consensus might invite horizontal collusion, i.e., experts might collude to avoid making conflicting reports. Such contracts are also exposed to vertical collusion: The principal may be tempted to bribe an expert into making a report that conflicts with the other expert's so as to reduce the latter's compensation. Note that horizontal collusion will arise naturally when communication between experts cannot be restricted, and vertical collusion when the principal cannot commit to publicly release the experts' reports.

When experts choose jointly whether to gather information (ex ante horizontal collusion), the two-expert organization remains optimal. When experts can coordinate their reports after having gathered information (ex post horizontal collusion), either organization form can be optimal depending on conditions we characterize. When only vertical collusion is possible, collusion-proof contracts are own-report-based, i.e., contingent on the outcome and the expert's own report but not on the other expert's. In that case, the two-expert organization dominates the one-expert organization, but agency costs imply only constant returns to scale. Finally, when both horizontal and vertical collusion are possible, collusion-proof contracts are outcome-based, and the organization forms' ranking is reversed: The one-expert organization is optimal.

Summarizing, the structure of agency costs for delegated expertise calls for using multiple experts. However, the benefits of such an organization depend on the scope for vertical and horizontal collusion. Without public and transparent reporting, relying on multiple experts may even be counterproductive. The one-expert organization's optimality is striking. While horizontal collusion allows two experts to behave as one vis-à-vis the principal, the two-expert organization is vulnerable to vertical collusion, which is impossible in the one-expert organization.

The design of expertise is well studied in Administrative Law (Hermitte [16], Harrison [15]) and Accounting (Baiman and Demski [1]), but less so in Economics. One can organize the 
relevant Economics literature along the steps of our analysis.

First, we study agency costs for one expert collecting information for a principal. This builds on Lambert [27], Demski and Sappington [7] and Malcomson [33] who study this problem under fairly general information structures. Our information structure is less general with discrete signals, decisions, and outcomes. However, with the risk-neutrality and limited liability assumptions, it makes our model tractable, allowing an extension to two experts.

Second, we compare agency costs for the one-expert and the two-expert organizations. This issue is related to recent organization theories. The adverse selection literature stresses that when agents are privately informed about their costs of doing complementary tasks, rents can be lower under centralization, i.e., in the one-agent organization (e.g., Baron and Besanko [3,4], Gilbert and Riordan [13], Laffont and Martimort [24], Dequiedt and Martimort [9]). The moral hazard literature yields similar results: As one agent performing two independent tasks can be rewarded when both succeed, rents are less than for two agents (Laux [28]). Instead, specific features of delegated expertise imply diseconomies of scope due to agency costs. The multidimensional adverse selection problem that arises with one expert is harder to solve. ${ }^{1}$ This problem spills over onto the signal gathering stage, creating endogenous diseconomies of scope outweighing the endogenous economies of scope stressed in the moral hazard literature. ${ }^{2}$

Third, we study collusion. Ex post horizontal collusion increases the cost of eliciting conflicting signals from two experts. This contrasts with the pure adverse selection literature where horizontal collusion (under asymmetric information) can be avoided at no cost (Laffont and Martimort [26], Che and Kim [5]). While vertical collusion is relevant beyond expertise, it has not received a general treatment in the literature, to our knowledge.

In Dewatripont and Tirole [10], one signal can be misreported only as being positive, and the other only as being negative. They show that with decision-based contracts the incentives for gathering two signals interact, making the two-expert organization optimal. Our mechanism design approach shows how horizontal and vertical collusion endogenize outcome-based (rather than the coarser decision-based) contracts. Absent collusion, conflicting signals are nevertheless easier to elicit with two experts. With collusion, however, a single expert can be optimal.

Sections 2 and 3 set up and study the one-expert/one-signal case. Section 4 studies the oneexpert/two-signals case. We identify the optimal organization absent collusion (Section 5) and in various collusion environments (Section 6). Section 7 studies extensions. Section 8 concludes.

\footnotetext{
${ }^{1}$ See Mookherjee and Tsumagari [35] and Severinov [38], as well as Laffont and Martimort [25].

${ }^{2}$ In a pure moral hazard setting, with exogenous synergies between tasks, complementary (substitute) tasks should be centralized (separated) (Holmström and Milgrom [19,20], Macho-Stadler and Perez-Castrillo [32], Itoh [21], Ramakrishan and Thakor [37]). However, this incentive effect is also mixed with better risk sharing under centralization, an issue we avoid by assuming risk-neutrality.
} 


\section{A Model of Delegated Expertise}

Consider a decision-maker (principal) and an agent (expert) whose task is to gather information on a risky project. Based on this information, the principal decides whether to undertake the project. Both the principal and the expert are risk-neutral. The expert is unbiased, i.e., a priori indifferent about whether the project is undertaken and about its outcome, and responds only to monetary incentives. (Section 8 discusses biased experts and non-monetary incentives.) The project's payoff is $\bar{S}>0$ if it succeeds, and $\underline{S}<0$ otherwise. Denote $\nu$ the prior for a success.

By incurring a personal cost $\psi$, the expert observes a signal $\sigma \in\{\underline{\sigma}, \bar{\sigma}\}$. Denote by $\theta \in(1 / 2,1]$ the signal's precision defined as $\operatorname{Proba}(\bar{\sigma} \mid \bar{S})=\operatorname{Proba}(\underline{\sigma} \mid \underline{S})=\theta$. Hence, $\bar{\sigma}$ is "good news" and $\underline{\sigma}$ "bad news" about the project. If the project is undertaken, its outcome is observed. Otherwise, its outcome is not observed (Section 7.1 studies the case in which it is).

Denote $p(\sigma)$ the likelihood of $\sigma \in\{\underline{\sigma}, \bar{\sigma}\}$ and $\nu(\sigma)$ the probability of success conditional on $\sigma$. More generally, we denote $p(\omega)$ the probability of an event $\omega$. For example, $p(\bar{\sigma})=$ $\nu \theta+(1-\nu)(1-\theta)$ and $\nu(\bar{\sigma})=\frac{\theta \nu}{\nu \theta+(1-\nu)(1-\theta)}$. We assume that absent further information, undertaking the project is inefficient:

$$
\nu \bar{S}+(1-\nu) \underline{S}<0 .
$$

but that a favorable signal makes the project valuable:

$$
\nu(\bar{\sigma}) \bar{S}+(1-\nu(\bar{\sigma})) \underline{S}>0 .
$$

First-best decision rule: Once $\psi$ is sunk, the optimal decision rule is to undertake the project if and only if $\sigma=\bar{\sigma}$. Hence gathering information is valuable if this is cheap ( $\psi$ small) enough, and the signal precise ( $\theta$ large) enough. The project's expected net surplus is:

$$
p(\bar{\sigma})(\nu(\bar{\sigma}) \bar{S}+(1-\nu(\bar{\sigma})) \underline{S})-\psi=\theta \nu \bar{S}+(1-\theta)(1-\nu) \underline{S}-\psi \geq 0 .
$$

\section{Expertise and Incentives}

This section describes the incentive issues arising in the principal-expert relationship. Departing from the first-best, three contracting frictions are assumed.

\section{Assumption}

(i) Moral hazard: Whether the expert acquires information or not is not observable.

(ii) Limited liability: Transfers from the principal to the expert are non-negative.

(iii) Soft information: The signal $\sigma$ is not observable by other parties. 
Point (i) implies that the expert must be given incentives to gather information. Point (ii) implies that incentive provision is costly for the principal. Without limited liability, the expert could be made residual claimant for the project. In particular, if the project fails after a positive report, the expert would have to pay the principal. Instead, under limited liability, incentive provision requires leaving an information rent to the expert. Point (iii) implies that the expert has private information. The expert produces information, a non-verifiable and fully manipulable output. In particular, he can report having observed a signal even if he has not. This feature distinguishes expertise from most other productive activities since output is generally observable.

A contract for the expert consists of transfers from the principal, and decisions whether to undertake the project based on his report $\hat{\sigma}$ on the signal. The project being risky, the transfers can be lotteries conditional on its outcome. Such lotteries are useful only if the project is undertaken. Otherwise, a constant transfer suffices. Formally, a contract is a menu of lotteries on transfers and a vector of decisions depending on the expert's report $\hat{\sigma} \in\{\underline{\sigma}, \bar{\sigma}\}$.

We begin by characterizing the optimal contract implementing the first-best decision rule. Such a contract is summarized by the expert's transfers $t_{0}$ if the project is rejected, $\bar{t}$ if it is undertaken and succeeds, and $\underline{t}$ if it fails. To streamline the presentation, we assume that $\underline{t}=0$, which is clearly optimal (see the Appendix for a proof).

Absent the incentive problem, the ex post information asymmetry between the principal and the expert could be solved at no cost with a flat contract since the expert is a priori unbiased. A flat contract does not work, however, when the expert must be induced to gather information. Indeed, he would remain uninformed and report any signal. Therefore the expert must be motivated to collect a signal, and to report it accurately, i.e., the problem combines moral hazard ex ante and adverse selection ex post.

Summarizing, the expert should neither prefer reporting $\underline{\sigma}$ after having observed $\bar{\sigma}$, i.e.,

$$
\nu(\bar{\sigma}) \bar{t} \geq t_{0},
$$

nor reporting $\bar{\sigma}$ after having observed $\underline{\sigma}$, i.e.,

$$
t_{0} \geq \nu(\underline{\sigma}) \bar{t}
$$

The contract must also satisfy a moral hazard incentive constraint for the expert to gather information. The expert must not prefer remaining uninformed and reporting either $\underline{\sigma}$, i.e.,

$$
p(\bar{\sigma}) \nu(\bar{\sigma}) \bar{t}+p(\underline{\sigma}) t_{0}-\psi \geq t_{0}
$$

or $\bar{\sigma}$ (in both cases his belief remains $\nu$ ), i.e.,

$$
p(\bar{\sigma}) \nu(\bar{\sigma}) \bar{t}+p(\underline{\sigma}) t_{0}-\psi \geq \nu \bar{t} .
$$

These two constraints imply the adverse selection constraints (3) and (4). For intuition, suppose the expert prefers reporting $\underline{\sigma}$ even after observing $\bar{\sigma}$ (i.e., constraint (3) is violated). Then he 
would report $\underline{\sigma}$ irrespective of the signal. But if the expert is not going to use the signal there is no point his paying $\psi$ to collect it (in particular constraint (5) is violated). Similarly, if constraint (4) is violated then the expert would report $\bar{\sigma}$ regardless of the signal, in which case there is no point his paying $\psi$ to collect it (in particular constraint (6) is violated). ${ }^{3}$

The expert's participation and limited liability constraints $p(\bar{\sigma}) \nu(\bar{\sigma}) \bar{t}+p(\underline{\sigma}) t_{0}-\psi \geq 0$, $\bar{t} \geq 0, t_{0} \geq 0$ are always satisfied in the environments below and they are omitted henceforth.

To implement the first-best decisions at minimum cost, the principal solves:

$$
\min _{\left\{\bar{t}, t_{0}\right\}} p(\bar{\sigma}) \nu(\bar{\sigma}) \bar{t}+p(\underline{\sigma}) t_{0} \quad \text { subject to }(5) \text { and }(6) .
$$

Denote $\underline{t}^{1,1}, \bar{t}^{1,1}, t_{0}^{1,1}$ and $U^{1,1}$ the optimal transfers and rent.

Lemma 1 The optimal incentive contract implementing the first-best decision rule rewards (resp. punishes) the expert when he makes a positive report and the project succeeds (resp. fails). The optimal transfers are as follows:

$$
\underline{t}^{1,1}=0, \quad \bar{t}^{1,1}=\frac{\psi}{\nu(1-\nu)(2 \theta-1)}, \quad \text { and } \quad t_{0}^{1,1}=\frac{\psi}{(1-\nu)(2 \theta-1)} .
$$

The expert's rent is:

$$
U^{1,1}=\frac{\psi}{p(\bar{\sigma} \mid \bar{S})-p(\bar{\sigma})}=\frac{\psi}{(1-\nu)(2 \theta-1)}
$$

The contract is simple. If his report is positive, the expert is rewarded only if the project succeeds. This motivates him to learn and report a positive signal. The expert is also rewarded for a negative report, motivating him to learn and report a negative signal. This feature is usual in moral hazard settings: An agent's rewards should be linked with the outcomes that are most informative about his having exerted effort, here about his having gathered information.

The rent's expression is also intuitive. First, the principal controls the expert thanks to the signal's precision (here $p(\bar{\sigma} \mid \bar{S})$ and $p(\underline{\sigma} \mid \underline{S}))$ : Moral hazard is less of an issue when the agent's performance is less noisy. As $p(\bar{\sigma} \mid \bar{S})$ increases, a positive report is more closely linked to the project's success, the project's outcome tracks the expert's effort more closely and the agency cost is lower. In our model, a negative report implies that the project is not undertaken and its outcome not observed. Therefore, $p(\underline{\sigma} \mid \underline{S})$ is irrelevant to incentive provision. Second, incentives are less powerful when the expert can easily "guess" the signal to be positive without observing it, i.e., when $p(\bar{\sigma})$ is large. Hence, the rent increases with $\nu$.

Finally, since the optimal contract is such that both moral hazard constraints (5) and (6) are binding, the RHS of these two constraints must be equal. Hence we get the following property which will be important to derive that of decreasing returns to scale due to agency costs.

\footnotetext{
${ }^{3}$ Demski and Sappington [7] discuss a model with information gathering (ex ante moral hazard) and production (ex post moral hazard) where productive effort is costless. They show that the latter incentive problem does not add any incentive cost in the case of two outcomes of the productive activity. Our result that adverse selection ex post is not a binding constraint is similar.
} 
Corollary 1 For one expert to gather one signal, the optimal contract is such that if the expert remained uninformed, he would be indifferent between reporting $\bar{\sigma}$ and $\underline{\sigma}$, i.e., $\nu \bar{t}^{1,1}=t_{0}^{1,1}$.

Decision rule: Having characterized the optimal contract implementing the first-best decision rule, we now optimize the rule itself. We focus on rules making an ex post optimal use of the information available. (Section 7.2 studies ex post inefficient rules). Here, the only relevant alternative to the first-best is never to do the project, which yields a zero payoff. Indeed, from condition (1), this dominates always doing the project. ${ }^{4}$ Agency costs reduce the value of gathering information: The principal will not hire an expert and will always reject the project unless the signal's value exceeds the sum of the cost $\psi$ and the agency cost, i.e., unless

$$
W^{1,1}=\theta \nu \bar{S}+(1-\theta)(1-\nu) \underline{S}-\psi-U^{1,1}>0 .
$$

\section{One-Expert/Two-Signals}

We show that with one expert collecting two signals, decreasing returns to scale obtain.

\subsection{Model}

Assume now that the expert can gather simultaneously two signals, $\left(\sigma_{1}, \sigma_{2}\right) \in\{\underline{\sigma}, \bar{\sigma}\}^{2}$, that are independently distributed, and have the same precision $\theta$ and $\operatorname{cost} \psi$. (Section 8 discusses the sequential case). Hence the cost of gathering two signals is twice that of gathering one signal, i.e., we assume constant returns to scale for the information gathering technology.

Denote $p\left(\sigma_{1}, \sigma_{2}\right)$ the probability of observing $\left(\sigma_{1}, \sigma_{2}\right)$ and $\nu\left(\sigma_{1}, \sigma_{2}\right)$ the conditional probability of success, e.g., $p(\bar{\sigma}, \bar{\sigma})=\theta^{2} \nu+(1-\theta)^{2}(1-\nu)$ and $\nu(\bar{\sigma}, \bar{\sigma})=\frac{\theta^{2} \nu}{\theta^{2} \nu+(1-\theta)^{2}(1-\nu)}$.

Doing the project is efficient only if both signals are positive. Indeed, with conflicting signals, Bayesian updating leaves the prior unchanged (i.e. $\nu(\bar{\sigma}, \underline{\sigma})=\nu$ ), and the status quo is efficient.

First-best decision rule: Gathering two signals is efficient if:

$$
-\theta(1-\theta)(\nu \bar{S}+(1-\nu) \underline{S}) \geq \psi
$$

The LHS is the benefit of a second signal: rejecting the project when the second signal contradicts a first positive one, i.e., with probability $p(\bar{\sigma}, \underline{\sigma})=\theta(1-\theta)$. The RHS is the cost of that signal.

\subsection{Diseconomies of Scale due to Agency Costs}

An incentive scheme specifies a decision rule and transfers depending on the project's outcome and both reports $\hat{\sigma}_{1}$ and $\hat{\sigma}_{2}$. However, not all such contracts are feasible. Indeed, when at least

\footnotetext{
${ }^{4}$ In this section, we consider a one-dimensional signal, reducing this set means never undertaking the project and increasing this set means always undertaking the project. Section 4 shows than when the expert gathers a multidimensional signal, the decision rule can be distorted in a less trivial way. See also Malcomson [33].
} 
one report is negative, the status quo is efficient. Hence all transfers when $\hat{\sigma}_{1}=\underline{\sigma}$ or $\hat{\sigma}_{2}=\underline{\sigma}$ must be equal. Otherwise, the expert would always make the report yielding the highest transfer.

Lemma 2 With one expert gathering two signals, contracts must be outcome-based, i.e., transfers are contingent only on the project's outcome (success/failure/rejection), not on the reports. ${ }^{5}$

Denote $t_{0}$ the common transfer for all report pairs yielding the status quo, and $\bar{t}$ that if the project is undertaken and succeeds. Three other adverse selection incentive constraints must be considered. First, the expert should prefer reporting $(\bar{\sigma}, \bar{\sigma})$ when this is what he observed,

$$
\nu(\bar{\sigma}, \bar{\sigma}) \bar{t} \geq t_{0}
$$

Conversely, the expert should not prefer reporting $(\bar{\sigma}, \bar{\sigma})$ after observing conflicting signals,

$$
t_{0} \geq \nu(\bar{\sigma}, \underline{\sigma}) \bar{t}
$$

or two negative signals,

$$
t_{0} \geq \nu(\underline{\sigma}, \underline{\sigma}) \bar{t}
$$

The expert should not prefer remaining uninformed:

$$
p(\bar{\sigma}, \bar{\sigma}) \nu(\bar{\sigma}, \bar{\sigma}) \bar{t}+(1-p(\bar{\sigma}, \bar{\sigma})) t_{0}-2 \psi \geq \max \left\{t_{0}, \nu \bar{t}\right\},
$$

which can easily be shown to imply constraints (11) and (13). Compared to the one-signal case, the expert has the new alternative to gather and base his report on only one signal, hence the new moral hazard incentive constraint: ${ }^{6}$

$$
p(\bar{\sigma}, \bar{\sigma}) \nu(\bar{\sigma}, \bar{\sigma}) \bar{t}+(1-p(\bar{\sigma}, \bar{\sigma})) t_{0}-2 \psi \geq p(\bar{\sigma}) \nu(\bar{\sigma}) \bar{t}+p(\underline{\sigma}) t_{0}-\psi \text {. }
$$

We now solve the principal's problem below:

$$
\min _{\left\{\bar{t}, t_{0}\right\}} p(\bar{\sigma}, \bar{\sigma}) \nu(\bar{\sigma}, \bar{\sigma}) \bar{t}+(1-p(\bar{\sigma}, \bar{\sigma})) t_{0} \quad \text { subject to }(12),(14) \text { and (15). }
$$

Let $\underline{t}^{1,2}, \vec{t}^{1,2}, t_{0}^{1,2}$ and $U^{1,2}$ denote the optimal transfers and rent.

Lemma 3 For one expert to gather two signals, the optimal incentive contract is:

$$
\underline{t}^{1,2}=0, \quad \frac{\nu \theta(2-\theta)}{1-p(\underline{\sigma}, \underline{\sigma})} \bar{t}^{1,2}=t_{0}^{1,2}=\frac{(2-\theta) \psi}{(1-\nu)(2 \theta-1)(1-\theta)} .
$$

\footnotetext{
${ }^{5}$ This property does not hold in more general models. See Section 7.1.

${ }^{6}$ The RHS should be $p(\bar{\sigma}) \max \left\{t_{0}, \nu(\bar{\sigma}) \bar{t}\right\}+p(\underline{\sigma}) \max \left\{t_{0}, \nu(\underline{\sigma}) \bar{t}\right\}-\psi$. However, constraint (14) supersedes both the case in which $t_{0}>\nu(\bar{\sigma}) \bar{t}$ and that in which $t_{0}<\nu(\underline{\sigma}) \bar{t}$. Indeed, if $t_{0}>\nu(\bar{\sigma}) \bar{t}$ this expression is equal to $t_{0}$ which is the first argument on the RHS of constraint (14) and if $t_{0}<\nu(\underline{\sigma}) \bar{t}$, it is equal to $\nu \bar{t}$ which is the second argument on the RHS of constraint (14). Note also that the expert's moral hazard and limited liability constraints imply his participation constraint, $p(\bar{\sigma}, \bar{\sigma}) \nu(\bar{\sigma}, \bar{\sigma}) \bar{t}+(1-p(\bar{\sigma}, \bar{\sigma})) t_{0}-2 \psi \geq 0$.
} 
The logic is as in the one-signal case. The expert is rewarded for reporting good news only if it is confirmed by the project's success. The status quo is also rewarded to elicit bad news.

Proposition 1 The expert's rent in the optimal contract is:

$$
U^{1,2}=\underbrace{\frac{\psi}{p(\bar{\sigma} \mid \bar{S})-p(\bar{\sigma})}}_{\text {Infra-marginal rent }}+\underbrace{\frac{\psi}{p(\bar{\sigma})(p(\bar{\sigma} \mid \bar{S}, \bar{\sigma})-p(\bar{\sigma} \mid \bar{\sigma}))}}_{\text {Marginal rent }}=\left(1+\frac{1}{1-\theta}\right) U^{1,1} .
$$

Delegation to one expert implies diseconomies of scale due to agency costs: $U^{1,2}>2 U^{1,1}$.

The problem of gathering two signals can be decomposed into that of gathering a first (inframarginal) signal, and that of gathering a second (marginal) one. For the expert to gather the first signal, he must be given the infra-marginal rent $U^{1,1}$ as in the one-signal case. This is the first term of the expression. To understand the second term, consider now the expert having gathered (but not yet observed) one signal and deciding whether to collect another. If the first signal turns out to be negative, the project is rejected irrespective of the second signal. Hence the second signal is pivotal only if the first one is positive, which occurs with probability $p(\bar{\sigma})$. Given this signal, the incentive problem is as in the one-signal case except that the probabilities must be conditioned on $\bar{\sigma}$.

The rent for the marginal signal exceeds that for the infra-marginal signal for two reasons. First, the second signal is useful only with probability $p(\bar{\sigma})$. Second, the rent decreases with how informative success is about the expert having observed $\bar{\sigma}$. But conditional on having observed $\sigma_{1}=\bar{\sigma}$, success is less informative about $\sigma_{2}$, i.e., $p(\bar{\sigma} \mid \bar{S}, \bar{\sigma})-p(\bar{\sigma} \mid \bar{\sigma})<p(\bar{\sigma} \mid \bar{S})-p(\bar{\sigma})$. Agency costs of inducing an expert to gather two signals are strictly more than twice that of inducing him to gather only one signal. Hence, agency costs imply diseconomies of scale.

This result being central to our analysis, we develop its intuition. Consider moral hazard incentive constraint (15), i.e., the expert must prefer collecting two signals rather than one.

Step 1: Absent constraints (12) and (15), the two-signal model is only a special case of the one-signal model. Indeed, the two-dimensional signal $\Sigma=\left(\sigma_{1}, \sigma_{2}\right)$ takes only two relevant values: either $\Sigma=\bar{\Sigma}=(\bar{\sigma}, \bar{\sigma})$ and the project is undertaken, or $\Sigma=\underline{\Sigma} \in\{(\bar{\sigma}, \underline{\sigma}),(\underline{\sigma}, \bar{\sigma}),(\underline{\sigma}, \underline{\sigma})\}$ and it is rejected. Hence, the two-signal model maps into the one-signal model, with cost $2 \psi$ rather than $\psi$, and precisions $p(\bar{\Sigma} \mid \bar{S})=\theta^{2}$ and $p(\underline{\Sigma} \mid \underline{S})=1-(1-\theta)^{2}$ rather than $\mathrm{p}(\bar{\sigma} \mid \bar{S})=\mathrm{p}(\underline{\sigma} \mid \underline{S})=\theta$. In that case, constraint (14) maps into constraints (5) and (6) and the rent is as in expression (8):

$$
\frac{2 \psi}{p(\bar{\Sigma} \mid \bar{S})-p(\bar{\Sigma})}=\frac{2 \psi}{(1-\nu)(2 \theta-1)}=2 U^{1,1}
$$

Moreover, by Corollary 1, constraint (12) is satisfied. In conclusion, absent constraint (15) constant returns to scale would obtain. 
Step 2: Consider the optimal contract absent constraint (15). Suppose the expert has collected (but not yet observed) $\sigma_{1}$ and is wondering whether to collect $\sigma_{2}$ or extrapolate from $\sigma_{1}$ and report $\Sigma=\left(\sigma_{1}, \sigma_{1}\right)$, i.e., report $\bar{\Sigma}$ if $\sigma_{1}=\bar{\sigma}$ and $\underline{\Sigma}$ if $\sigma_{1}=\underline{\sigma}$. The only reason for collecting $\sigma_{2}$ is if this sometimes leads him to change his report. If $\sigma_{2}=\sigma_{1}$, the expert sticks to $\Sigma=\left(\sigma_{1}, \sigma_{1}\right)$. If $\sigma_{2} \neq \sigma_{1}$, the expert is back to his prior belief (i.e., $\nu(\bar{\sigma}, \underline{\sigma})=\nu$ ), in which case he is indifferent between reporting $\bar{\Sigma}$ or $\underline{\Sigma}$ (Corollary 1$)$ and might as well stick to $\Sigma=\left(\sigma_{1}, \sigma_{1}\right)$. But if the expert is not going to use $\sigma_{2}$, there is no point paying $\psi$ to collect it. Hence, constraint (15) is violated, which implies decreasing returns to scale.

This intuition can be generalized. In particular, the decreasing returns to scale property does not rely on the outcome not being observed if the project is rejected (see Section 7.1).

Decision rule: The principal induces the expert to gather a signal when its value exceeds the sum of the cost $\psi$ and the agency cost. Therefore, he induces the expert to gather (at least) one signal if condition (9) holds, and also a second one only if

$$
-\theta(1-\theta)(\nu \bar{S}+(1-\nu) \underline{S}) \geq \psi+\frac{U^{1,1}}{1-\theta} .
$$

Hence an optimal way of reducing agency costs can involve distorting the decision rule.

Corollary 2 With one expert, the principal can find it optimal to distort the decision rule by having the expert gather only one signal even if two signals are first-best optimal.

\section{Two-Experts/Two-Signals: No Collusion}

Consider now two experts, $A$ and $B$, privately observing one signal each. We focus on the case where the experts make simultaneous reports, not knowing the other's effort, signal, or report. The main result is that the principal's ability to cross-check the experts' reports implies increasing returns to scale, which in turn means that the two-expert organization is optimal.

\subsection{Optimal Contracts}

Without loss of generality, we assume that both experts receive the same contract. Each expert receives $\bar{t}$ if the project succeeds. Note that, unlike in the one-expert case, transfers can depend on how the status quo is reached: With conflicting reports, the expert reporting good (resp. bad) news receives $t_{G}\left(\right.$ resp. $\left.t_{B}\right)$, and each expert receives $t_{0}$ when both news are bad.

When deviating from the proposed equilibrium strategy, each expert believes that the other one sticks to the obedient equilibrium strategy. Hence the Bayesian adverse selection incentive compatibility constraint if an expert observes $\bar{\sigma}$ is:

$$
p(\bar{\sigma} \mid \bar{\sigma}) \nu(\bar{\sigma}, \bar{\sigma}) \bar{t}+p(\underline{\sigma} \mid \bar{\sigma}) t_{G} \geq p(\bar{\sigma} \mid \bar{\sigma}) t_{B}+p(\underline{\sigma} \mid \bar{\sigma}) t_{0} .
$$


When the expert observes $\underline{\sigma}$, the constraint is:

$$
p(\bar{\sigma} \mid \underline{\sigma}) t_{B}+p(\underline{\sigma} \mid \underline{\sigma}) t_{0} \geq p(\bar{\sigma} \mid \underline{\sigma}) \nu(\underline{\sigma}, \bar{\sigma}) \bar{t}+p(\underline{\sigma} \mid \underline{\sigma}) t_{G} .
$$

An expert's moral hazard incentive constraint is now:

$$
p(\bar{\sigma}, \bar{\sigma}) \nu(\bar{\sigma}, \bar{\sigma}) \bar{t}+p(\bar{\sigma}, \underline{\sigma})\left(t_{G}+t_{B}\right)+p(\underline{\sigma}, \underline{\sigma}) t_{0}-\psi \geq \max \left\{p(\bar{\sigma}) \nu(\bar{\sigma}) \bar{t}+p(\underline{\sigma}) t_{G}, p(\bar{\sigma}) t_{B}+p(\underline{\sigma}) t_{0}\right\} .
$$

The adverse selection incentive constraints are implied by those of moral hazard, and are omitted henceforth, as is each expert's participation constraint $p(\bar{\sigma}, \bar{\sigma}) \nu(\bar{\sigma}, \bar{\sigma}) \bar{t}+p(\bar{\sigma}, \underline{\sigma})\left(t_{G}+t_{B}\right)+$ $p(\underline{\sigma}, \underline{\sigma}) t_{0}-\psi \geq 0$. Finally, the relevant limited liability constraints are:

$$
t_{G}, t_{B} \geq 0
$$

The principal's program is:

$$
\min _{\left\{\bar{t}, t_{G}, t_{B}, t_{0}\right\}} p(\bar{\sigma}, \bar{\sigma}) \nu(\bar{\sigma}, \bar{\sigma}) \bar{t}+p(\bar{\sigma}, \underline{\sigma})\left(t_{G}+t_{B}\right)+p(\underline{\sigma}, \underline{\sigma}) t_{0} \quad \text { subject to }(20) \text { and }(21) .
$$

Denote $\underline{t}^{2,2}, t_{G}^{2,2}, t_{B}^{2,2}, \vec{t}^{2,2}, t_{0}^{2,2}$ and $U^{2,2}$ the optimal transfers and rent.

Lemma 4 Absent collusion, for two experts to gather one signal each, the optimal symmetric incentive contract is report-based, i.e., transfers are contingent not only on the project's outcome but also on the reports. It punishes experts for conflicting reports. The optimal transfers are:

$$
\underline{t}^{2,2}=t_{G}^{2,2}=t_{B}^{2,2}=0, \quad \vec{t}^{2,2}=\frac{p(\underline{\sigma}) \psi}{\nu(1-\nu)(2 \theta-1) \theta^{2}}, \quad \text { and } \quad t_{0}^{2,2}=\frac{\psi}{(1-\nu)(2 \theta-1) \theta} .
$$

As in the one-expert/one-signal case, the principal can use the project's outcome to check a positive report: Following such a report, the expert is rewarded only when the project (is undertaken and) succeeds $\left(\underline{t}^{2,2}=t_{G}^{2,2}=0\right)$. Now, however, the principal has an instrument to check a negative report as well: Because the signals are correlated, following a negative report, the expert is rewarded only when the other expert's report is also negative $\left(t_{B}^{2,2}=0\right) .{ }^{7}$

\subsection{Economies of Scale due to Agency Costs}

The principal's ability to cross-check the experts' reports allows him to motivate each expert at a lower cost than in the one-expert/one-signal case.

Proposition 2 Assume that collusion is not possible.

- In the optimal contract, each expert's rent is:

$$
U^{2,2}=\frac{\psi}{(p(\bar{\sigma} \mid \bar{S})-p(\bar{\sigma}))+\left(p\left(\sigma_{1}=\underline{\sigma} \mid \sigma_{2}=\underline{\sigma}\right)-p\left(\sigma_{1}=\underline{\sigma}\right)\right)}=\frac{p(\underline{\sigma}) \psi}{(1-\nu)(2 \theta-1) \theta} .
$$

\footnotetext{
${ }^{7}$ This is essentially an application of Holmström [18] and Mookherjee [34].
} 
- Delegation to two experts implies economies of scale due to agency costs: $2 U^{2,2}<2 U^{1,1}$.

- The two-expert organization is optimal: $2 U^{2,2}<U^{1,2}$.

The rent is as in the one-expert/one-signal case, except that now the principal can use not only the correlation between $\bar{\sigma}$ and $\bar{S}$ to check the expert's report if it is positive, but also that between $\sigma_{1}=\underline{\sigma}$ and $\sigma_{2}=\underline{\sigma}$ to check the expert's report if it is negative. Note that thanks to this second instrument, each expert's rent is less than in the one-expert/one-signal case, i.e., there are economies of scale due to agency costs. This implies that the two-expert dominates the one-expert organization because $2 U^{2,2}<2 U^{1,1}<U^{1,2}$.

Note that report-based contracts have two more degrees of freedom than outcome-based contracts: Neither $t_{G}$ nor $t_{B}$ need equal $t_{0}$. The optimal contract uses both degrees of freedom, but for different reasons. Setting $t_{G}=0$ allows the principal to focus the reward of an expert reporting $\bar{\sigma}$ on the state that is most informative about his being informed, i.e., the project's success. Since the principal can also do so in the one-expert/one-signal case, this is not generating increasing returns to scale, i.e., under the constraint that $t_{B}=t_{0}$, constant returns to scale would obtain. Setting $t_{B}=0$ allows the principal to cross-check the experts' negative reports. It is this instrument, unavailable in the one-expert/one-signal case, that generates economies of scale.

Decision rule: With two experts, the principal prefers having two signals if:

$$
W^{2,2}=\theta^{2} \nu \bar{S}+(1-\theta)^{2}(1-\nu) \underline{S}-2 \psi-2 U^{2,2}>W^{1,1} .
$$

Agency costs being less than with a single expert, the principal relies more often on a second signal when deciding whether to undertake the project.

Corollary 3 Absent collusion, the principal finds it less often optimal to distort the decision rule by relying on a single signal than when only one expert is available.

\section{Collusion and the Organization of Delegated Expertise}

We now introduce the possibility of ex ante and ex post horizontal collusion between experts, and of vertical collusion between the principal and each expert. We characterize the optimal organization of delegated expertise in different collusion environments.

\subsection{Ex ante Horizontal Collusion}

A problem with the optimal report-based contract is that it admits other less desirable equilibria. If expert $B$ remains uninformed and reports bad news, expert $A$ 's best response is to do the same. Indeed, even if he observed a good signal $A$ would never report it since conflicting reports 
are punished. As a result, $A$ is better off remaining uninformed and reporting bad news. Hence an equilibrium exists in which both experts always report bad news, and get a payoff $t_{0}^{2,2}$ that strictly exceeds $U^{2,2}$. Similarly, another equilibrium exists in which experts always report good news. In this equilibrium, each expert's payoff, $\nu \vec{t}^{2,2}$, exceeds $U^{2,2}$ but is less than $t_{0}^{2,2}$. Note that in both these equilibria the principal's payoff is negative. In the first one, although the project is never undertaken, the principal bears the cost of paying the experts. In the second one, he also bears that of undertaking a negative-value project.

Although reached non-cooperatively, these outcomes can be viewed as collusive since the experts are better off than by behaving obediently and truthfully. ${ }^{8}$ Hence, following most of the literature, we look for contracts immune to non-obedient and non-truthful pure strategy equilibria. For brevity, we only report the main results of this analysis (developed in the Appendix).

As the two experts's contracts can differ, we denote $U^{i *}$ the optimal rent of expert $i \in\{A, B\}$.

Proposition 3 To implement the first-best decision rule under ex ante horizontal collusion:

- The optimal collusion-proof contract is asymmetric between the experts whose rents are:

$$
U^{A *}=\frac{\psi}{p(\bar{\sigma} \mid \bar{S}, \bar{\sigma})-p(\bar{\sigma} \mid \bar{\sigma})} \quad \text { and } \quad U^{B *}=U^{2,2}
$$

- The two-expert organization is optimal: $U^{A *}+U^{B *}<U^{1,2}$.

The intuition is simple. The principal must induce at least one expert to deviate from the putative collusive equilibria in which the experts remain uninformed and report $(\underline{\sigma}, \underline{\sigma}),(\bar{\sigma}, \bar{\sigma})$, $(\bar{\sigma}, \underline{\sigma})$ or $(\underline{\sigma}, \bar{\sigma})$. Because the optimal contract absent collusion punishes conflicting reports, it will induce expert $B$ to deviate from $(\bar{\sigma}, \underline{\sigma})$ and $(\underline{\sigma}, \bar{\sigma})$ irrespective of expert $A$ 's contract. Inducing expert $A$ to deviate from $(\underline{\sigma}, \underline{\sigma})$ requires setting $t_{0}<t_{G}$ which optimally achieved by setting $t_{0}=0$ and $t_{G}>0$ arbitrarily small. But now expert $A$ is rewarded only conditional on expert $B$ reporting $\bar{\sigma}$, hence the expression of $U^{A *}$.

Now recall that $U^{1,2}$ is the sum of the rent for an infra-marginal signal and that for a marginal one. Since $U^{B *}$ is as in the no-collusion case, it is less than the rent for an infra-marginal signal. Note that $U^{A *}$ is less than the rent for the marginal signal (i.e. it is that rent times $p(\bar{\sigma})$ ). Indeed, in both cases the expert is rewarded only conditional on the other signal being $\bar{\sigma}$. However, here the fact that incurring $\psi$ is useful only with probability $p(\bar{\sigma})$ does not increase the rent because the principal can simply scale up the transfers. Hence $U^{A *}=\frac{\psi}{(1-\nu(\bar{\sigma})(2 \theta-1)}$. This is not possible in the one-expert/two-signal case without distorting the incentive to collect the infra-marginal signal, and the corresponding rent is $\frac{\psi}{(1-\nu(\bar{\sigma}))(2 \theta-1)}$. Overall, this implies that $U^{A *}+U^{B *}<U^{1,2}$.

\footnotetext{
${ }^{8}$ Demski and Sappington [6] present an adverse selection model with a cross-checking mechanism not immune to collusive (non-cooperative) outcomes which are non-truthful. Our non-cooperative equilibrium comes from the moral hazard stage.
} 


\subsection{Ex post Horizontal Collusion}

Consider now ex post horizontal collusion: Having collected signals, experts can jointly manipulate their reports and exchange side-transfers. This type of collusion arises when communication between the experts cannot be controlled perfectly by the principal. Following the literature, we assume that experts can credibly share their information and collude by making their jointly optimal reports and exchanging side-transfers (Baron and Besanko [4] calls a similar assumption internally verifiable information) and that side-contracts are enforceable (Tirole [39]). ${ }^{9}$

Once the experts have gathered the signals, they make reports maximizing their joint-payoff. Therefore, a contract is robust to ex post horizontal collusion between the experts if and only if it satisfies the following horizontal coalition-incentive constraints: ${ }^{10}$

$$
\begin{aligned}
2 t_{0} & =t_{G}+t_{B}, \\
\nu(\bar{\sigma}, \bar{\sigma}) \bar{t} & \geq t_{0} \\
t_{0} & \geq \nu \bar{t} .
\end{aligned}
$$

Constraint (25) reflects the principal's inability to distinguish between all pairs of reports yielding the status quo. Given constraint (25), constraint (26) means that the experts must be jointly better off recommending to undertake the project when they have observed two positive signals. Similarly, given constraint (25), constraint (27) means that the experts must be jointly better off recommending to reject the project when they have observed at least one negative signal. Clearly, the optimal report-based contract of Lemma 4 violates constraint (25).

For brevity, we only report the main results of this analysis (developed in the Appendix).

Proposition 4 Under ex post horizontal collusion, $\forall \nu \in[0,1], \exists \theta^{*}(\nu) \in[1 / 2,1]$ with $\theta^{*}(\nu)$ strictly increasing with $\nu, \theta^{*}(0)>1 / 2$ and $\theta^{*}(1)=1$ and such that:

- For $\theta \leq \theta^{*}(\nu)$, the one-expert organization is optimal.

- For $\theta \geq \theta^{*}(\nu)$, the two-expert organization is optimal.

\subsection{Vertical Collusion}

Consider now vertical collusion between the principal and each expert. If both reports are negative, the principal should pay $t_{0}$ to each expert. He would be better off bribing one expert into switching to a positive report, and thus avoid paying the other expert.

\footnotetext{
${ }^{9}$ Credible disclosure may stem from a fellow expert being less easily fooled than the principal. Moreover experts have incentives to disclose their signals truthfully to each other in any report-based contract punishing conflicting reports. This may also justify our focus on credible disclosure to analyze collusion in more general mechanisms. With credible disclosure, we characterize an upper bound on the effect of ex post horizontal collusion.

${ }^{10}$ These conditions are easily derived from the coalition incentive constraints: $2 \nu(\bar{\sigma}, \bar{\sigma}) \bar{t} \geq \max \left\{t_{1}+t_{2}, 2 t_{0}\right\}$, $2 t_{0} \geq \max \left\{t_{1}+t_{2}, 2 \nu(\underline{\sigma}, \underline{\sigma}) \bar{t}\right\}$, and $t_{1}+t_{2} \geq \max \left\{2 t_{0}, 2 \nu \bar{t}\right\}$. Note also that the experts' side payments satisfy their limited liability constraints.
} 
Note that vertical collusion is relevant only when it leaves the decision unchanged. Given a contract, vertical collusion might lead to a decision change. However, such a contract would never be considered in the first place, i.e., absent collusion, as the principal would be better off with a single signal or none. As a corollary, vertical collusion is relevant only when the project must be rejected: When both signals are positive, changing a report changes the decision.

A contract is robust to vertical collusion between the principal and each expert if and only if it is own-report-based, i.e., satisfies the following vertical coalition incentive constraint:

$$
t_{B}=t_{0} .
$$

We denote $U^{2,2, v}$ each expert's rent in the optimal contract.

Proposition 5 Under vertical collusion:

- Delegation to two experts implies constant returns to scale due to agency costs: $U^{2,2, v}=$ $U^{1,1}$.

- The two-expert organization is optimal: $2 U^{2,2, v}<U^{1,2}$.

In the no-collusion case, the increasing returns to scale stem from the principal being able to cross-check the experts' negative signals, which requires setting $t_{B} \neq t_{0}$ (see Section 5 ). Absent this possibility, the principal cannot provide each expert with more powerful incentives than in the one-expert/one-signal model. Vertical collusion rules out cross-checking and the associated increasing returns, and only constant returns obtain. Since vertical collusion arises when the principal cannot publicly release the experts' reports, our analysis implies that increasing returns to scale rely on the principal's ability to publicize the experts' reports.

\subsection{Vertical and Horizontal Collusion: Outcome-Based Contracts}

Finally, assume that both ex post horizontal and vertical collusion are possible. This case is relevant since, once vertical collusion has been fought and the principal publicly releases the experts' reports, they can coordinate their messages ex post.

From conditions (25) and (28), a contract is robust to ex post horizontal and vertical collusion if and only if it is outcome-based, i.e.,

$$
t_{0}=t_{G}=t_{B}
$$

Denote $\underline{t}^{2,2, v h}, t_{G}^{2,2, v h}, t_{B}^{2,2, v h}, \bar{t}^{2,2, v h}, t_{0}^{2,2, v h}$ and $U^{2,2, v h}$ the optimal transfers and rent.

Proposition 6 Under ex post horizontal and vertical collusion, the optimal collusion-proof contract implementing the first-best decision rule is such that:

$$
\vec{t}^{2,2, v h}=\frac{\psi / p(\bar{\sigma})}{\nu(\bar{\sigma})(1-\nu(\bar{\sigma}))(2 \theta-1)} \quad \text { and } t_{0}^{2,2, v h}=\frac{\psi / p(\bar{\sigma})}{(1-\nu(\bar{\sigma}))(2 \theta-1)} .
$$


Each expert's rent is:

$$
U^{2,2, v h}=\frac{\psi}{p(\bar{\sigma})(p(\bar{\sigma} \mid \bar{S}, \bar{\sigma})-p(\bar{\sigma} \mid \bar{\sigma}))}=\frac{1}{1-\theta} U^{1,1}
$$

The one-expert organization is optimal: $2 U^{2,2, v h}>U^{1,2}$.

Each expert's rent corresponds to the second term in expression (16)'s RHS, i.e., the rent left to a single expert for the marginal signal. The intuition is as follows. First note that the contract space, i.e., that of outcome-based contracts, is the same as in the one-expert/two-signals case. Second, with two experts and outcome-based contracts, in equilibrium, each expert expects the other one to gather and report his signal. Therefore, each of the two experts behaves as if he were gathering the marginal signal, and must be given the corresponding rent.

Recall the decreasing returns to scale property of the one-expert/two-signal case. The rent needed for the expert to gather and report the marginal signal exceeds that for the infra-marginal signal. Since both experts need to be given the larger rent corresponding to the marginal signal, the ranking of organizations obtained absent collusion (Proposition 2) is reversed.

Outcome-based contracts although a priori attractive because immune to all forms of collusion, increase agency costs too much. However, using a single expert allows the principal to suppress the scope for vertical collusion. Note also that given vertical collusion, any departure from constant returns to scale can be seen as arising from the possibility of horizontal collusion, e.g., due to the principal's inability to control communication between experts.

\section{Extensions}

\subsection{Symmetric Case}

This section relaxes the assumption that if the project is rejected, its outcome is not observed. Instead, we assume that it is observed with an exogenous probability $\varepsilon>0 .{ }^{11}$ We refer to this model as the "symmetric" case because the outcome is (possibly) observed whether the project is undertaken or not, and to the model analyzed previously as the "asymmetric" case. The rest of the section proves the following result.

Proposition 7 Consider the symmetric case.

- In the two-experts/two-signal case, giving each expert the optimal contract of the oneexpert/one-signal case is an optimal contract absent collusion that is also robust to horizontal and/or vertical collusion.

\footnotetext{
${ }^{11}$ The analysis is unchanged if the two outcomes are observed with different probabilities.
} 
- The two-expert organization is optimal under (i) no collusion, (ii) ex ante horizontal collusion, (iii) ex post horizontal collusion, (iv) vertical collusion, and (v) both horizontal and vertical collusion.

\subsubsection{One-Expert/One-Signal}

A contract inducing the expert to collect and report the signal specifies transfers $t(\hat{\sigma}, X)$ where $X \in\{\varnothing, \underline{S}, \bar{S}\}$ is the project's outcome, $\varnothing$ denoting the case in which project is rejected and its outcome not observed. We assume (and prove in the Appendix) that all transfers but $t(\bar{\sigma}, \bar{S})$ and $t(\underline{\sigma}, \underline{S})$ are equal to zero. The intuition is as before: The expert's rewards should be linked with the outcomes most informative about his having gathered information. We can thus focus on two (expected) transfers which we denote $\bar{t} \equiv t(\bar{\sigma}, \bar{S})$ and $\underline{\tau} \equiv \varepsilon \times t(\underline{\sigma}, \underline{S})$.

The contract is subject to moral hazard incentive constraints:

$$
p(\bar{\sigma}) \nu(\bar{\sigma}) \bar{t}+p(\underline{\sigma})(1-\nu(\underline{\sigma})) \underline{\tau}-\psi \geq \max \{(1-\nu) \underline{\tau}, \nu \bar{t}\} .
$$

As before, these constraints imply the adverse selection, participation and limited liability constraints (omitted here), and are binding at the optimum.

Lemma 5 In the optimal contract for the one-expert/one-signal case, the expert's rent is:

$$
U^{1,1}=\nu \bar{t}^{1,1}=(1-\nu) \underline{\tau}^{1,1}=\frac{\psi}{(p(\bar{\sigma} \mid \bar{S})-p(\bar{\sigma}))+(p(\underline{\sigma} \mid \underline{S})-p(\underline{\sigma}))}=\frac{\psi}{2 \theta-1} .
$$

As in the asymmetric case, the principal can use the project's success to check a positive report. Here, however, he has a second instrument: He can also use the project's failure to check a negative report. Therefore, Lemma 5 simply generalizes expression (8).

\subsubsection{One-Expert/Two-Signals}

We show that the decreasing returns to scale still obtain, and extend our two-step intuition.

An incentive scheme must specify a decision rule and the expert's transfer depending on the project's outcome as well as his two reports $\hat{\sigma}_{1}$ and $\hat{\sigma}_{2}$, i.e., $t\left(\hat{\sigma}_{1}, \hat{\sigma}_{2}, X\right)$. As before, we assume that all transfers but $t(\bar{\sigma}, \bar{\sigma}, \bar{S}), t(\underline{\sigma}, \bar{\sigma}, \bar{S}), t(\underline{\sigma}, \bar{\sigma}, \underline{S})$, and $t(\underline{\sigma}, \underline{\sigma}, \underline{S})$ are equal to zero, and denote $\bar{t} \equiv t(\bar{\sigma}, \bar{\sigma}, \bar{S}), \tau_{1} \equiv \varepsilon \times t(\underline{\sigma}, \bar{\sigma}, \bar{S}), \tau_{2} \equiv \varepsilon \times t(\underline{\sigma}, \bar{\sigma}, \underline{S})$, and $\underline{\tau} \equiv \varepsilon \times t(\underline{\sigma}, \underline{\sigma}, \underline{S})$.

The expert should prefer reporting $(\bar{\sigma}, \bar{\sigma})$ if this is what he observed rather than reporting conflicting or two negative signals:

$$
\nu(\bar{\sigma}, \bar{\sigma}) \bar{t} \geq \max \left\{\nu(\bar{\sigma}, \bar{\sigma}) \tau_{1}+(1-\nu(\bar{\sigma}, \bar{\sigma})) \tau_{2},(1-\nu(\bar{\sigma}, \bar{\sigma})) \underline{\tau}\right\} .
$$

The contract must also satisfy two other similar adverse selection constraints:

$$
\nu \tau_{1}+(1-\nu) \tau_{2} \geq \max \{\nu \bar{t},(1-\nu) \underline{\tau}\}
$$


and

$$
(1-\nu(\underline{\sigma}, \underline{\sigma})) \underline{\tau} \geq \max \left\{\nu(\underline{\sigma}, \underline{\sigma}) \bar{t}, \nu(\underline{\sigma}, \underline{\sigma}) \tau_{1}+(1-\nu(\underline{\sigma}, \underline{\sigma})) \tau_{2}\right\} .
$$

Moreover, the expert must neither prefer remaining uninformed:

$p(\bar{\sigma}, \bar{\sigma}) \nu(\bar{\sigma}, \bar{\sigma}) \bar{t}+2 p(\bar{\sigma}, \underline{\sigma})\left(\nu \tau_{1}+(1-\nu) \tau_{2}\right)+p(\underline{\sigma}, \underline{\sigma})(1-\nu(\underline{\sigma}, \underline{\sigma})) \underline{\tau}-2 \psi \geq \max \left\{\nu \bar{t},(1-\nu) \underline{\tau}, \nu \tau_{1}+(1-\nu) \tau_{2}\right\}$

nor prefer gathering and basing his report on only one signal:

$$
\begin{gathered}
p(\bar{\sigma}, \bar{\sigma}) \nu(\bar{\sigma}, \bar{\sigma}) \bar{t}+2 p(\bar{\sigma}, \underline{\sigma})\left(\nu \tau_{1}+(1-\nu) \tau_{2}\right)+p(\underline{\sigma}, \underline{\sigma})(1-\nu(\underline{\sigma}, \underline{\sigma})) \underline{\tau}-2 \psi \geq \\
p(\bar{\sigma}) \nu(\bar{\sigma}) \bar{t}+p(\underline{\sigma})(1-\nu(\underline{\sigma})) \underline{\tau}-\psi, \\
\max \left\{\begin{array}{c}
p(\bar{\sigma}) \nu(\bar{\sigma}) \bar{t}+p(\underline{\sigma})\left(\nu(\underline{\sigma}) \tau_{1}+(1-\nu(\underline{\sigma})) \tau_{2}\right)-\psi, \\
p(\bar{\sigma})\left(\nu(\bar{\sigma}) \tau_{1}+(1-\nu(\bar{\sigma})) \tau_{2}\right)+p(\underline{\sigma})(1-\nu(\underline{\sigma})) \underline{\tau}-\psi
\end{array}\right\} .
\end{gathered}
$$

Lemma 6 In an optimal contract for the one-expert/two-signals case, the expert's rent is:

$$
\begin{aligned}
U^{1,2} & =\underbrace{\frac{\psi}{(p(\bar{\sigma} \mid \bar{S})-p(\bar{\sigma}))+(p(\underline{\sigma} \mid \underline{S})-p(\underline{\sigma}))}}_{\text {Infra-marginal rent }}+\underbrace{\frac{\psi}{p(\bar{\sigma})(p(\bar{\sigma} \mid \bar{S}, \bar{\sigma})-p(\bar{\sigma} \mid \bar{\sigma}))+p(\underline{\sigma})(p(\underline{\sigma} \mid \underline{S}, \underline{\sigma})-p(\underline{\sigma} \mid \underline{\sigma}))}}_{\text {Marginal rent }} \\
& =\left(1+\frac{1}{1-\theta}\right) U^{1,1} .
\end{aligned}
$$

Delegated expertise to one expert exhibits diseconomies of scale due to agency costs: $U^{1,2}>2 U^{1,1}$.

This result simply generalizes Proposition 1 and can be understood with a similar but more general two-step intuition. Ignore the moral hazard incentive constraint (36), i.e., the expert must prefer collecting two signals rather than one.

Step 1: Assume that the principal gives the expert two independent contracts for collecting the two signals, each contract being the optimal one for the one-expert/one-signal case, i.e., $\bar{t}=2 \bar{t}^{1,1}, \underline{\tau}=2 \underline{\tau}^{1,1}, \tau_{1}=\tau_{2}=\bar{t}^{1,1}+\underline{\tau}^{1,1}$. This scheme satisfies the adverse selection constraints (32) to (34). It also satisfies the moral hazard constraint (35) with equality, i.e., the expert is indifferent between gathering two signals and none (and making any report). Hence, absent constraint (36), the expert's rent would be $2 U^{1,1}$, i.e., there would be constant returns to scale.

Step 2: The optimal contract absent constraint (36) satisfies Corollary 1. Hence, as in the asymmetric case, the expert is never strictly better off using the second signal, and therefore will not pay $\psi$ to collect it: Constraint (36) is violated, which implies decreasing returns to scale.

\subsubsection{Two-Experts/Two-Signals}

We show that the increasing returns to scale property no longer holds. 
Lemma 7 Irrespective of the collusion environment, delegation to two experts implies constant returns to scale $\left(2 U^{2,2}=2 U^{1,1}\right)$ and the two-expert organization is optimal $\left(2 U^{2,2}<U^{1,2}\right)$.

The intuition for this result is simple. In our model, the information contained in an expert's report about the signal observed by the other expert is redundant if the project's outcome is observed. Hence, the principal need not condition an expert's (expected) reward on the other expert's report. He can simply give each expert the optimal contract of the one-expert/onesignal case. This rules out cross-checking and therefore increasing returns to scale. ${ }^{12}$ Finally, the optimal contracts being independent and implementing ex post efficient decision rules, there is no scope for horizontal or vertical collusion.

\subsubsection{Properties}

We conclude with two properties that will simplify the analysis in the next section. These can be proved by inspection of the agency costs in different cases.

Lemma 8 In all cases, the agency costs in the symmetric case $(\varepsilon>0)$ are (i) independent of $\varepsilon$ and (ii) strictly less than in the asymmetric case with ex post efficient decision rules $(\varepsilon=0)$.

Point (i) reflects the fact that the principal and the experts' payoffs (and therefore their incentives) depend on expected transfers contingent on report and outcome. The expected transfers can always be kept constant through a decrease in $\varepsilon$ by a corresponding scaling up of the transfers. Point (ii) reflects the fact that in all cases, the principal can use more or better instruments to check the experts' reports.

\subsection{Asymmetric Case: Ex post Inefficient Decision Rules}

Our analysis of the asymmetric case has focused on rules that use the information available efficiently. Beyond the usual motivation based on renegotiation-proofness, this focus allowed us to avoid distinguishing the effects of vertical collusion from those of renegotiation. However, a growing literature studies how committing to a distorted use of information can be part of an optimal incentive scheme for information gathering (e.g., Gerardi and Yariv [12]). In this section, we relax this assumption and characterize the optimal organization. We assume that the principal can commit to undertaking the project with probability $\varepsilon>0$ when it is efficient to reject it, $\varepsilon$ being a choice variable. ${ }^{13}$

\footnotetext{
${ }^{12}$ Note that if the signals' conditional distributions were not independent, cross-checking would remain useful and imply increasing returns to scale in the symmetric case, albeit less than in the asymmetric case.

${ }^{13}$ More generally, the project could be undertaken with different probabilities depending on the reports. We ignore this possibility for brevity. It is easily checked that this shortcut does not affect the results.
} 
Proposition 8 Consider the asymmetric case with ex post inefficient decision rules.

- In the two-experts/two-signals case, setting $\varepsilon>0$ arbitrarily close to zero and giving each expert the optimal contract of the one-expert/one-signal symmetric case is an optimal contract absent collusion that is also robust to horizontal collusion but not to vertical collusion.

- Under vertical collusion and both horizontal and vertical collusion, it is optimal to set $\varepsilon=0$ and to give the experts the contracts of the asymmetric case with ex post efficient decision rules.

- The two-expert organization is optimal under (i) no collusion, (ii) ex ante horizontal collusion, (iii) ex post horizontal collusion, (iv) vertical collusion. The one-expert organization is optimal under (v) both horizontal and vertical collusion.

For a given $\varepsilon>0$, the set of feasible contracts is as in the symmetric case where if the project is rejected, its outcome is observed with probability $\varepsilon>0$. Therefore the rent that must be left to the expert(s) is also as in the symmetric case. Hence, we can use Lemma 8 to understand the principal's optimization problem. Point (i) implies that if the principal is going to set $\varepsilon>0$, he should make it as small as possible so as to minimize inefficiencies. Doing so, the principal's payoff is arbitrarily close to that in the symmetric case. Point (ii) implies that the rent for $\varepsilon>0$ is always less than for $\varepsilon=0$. Taken together, these remarks imply that whenever possible, the principal will set $\varepsilon>0$ and arbitrarily close to zero. The rest of the analysis is straightforward. Absent collusion, the analysis is as in the symmetric case. Since horizontal collusion does not preclude $\varepsilon>0$, its analysis is again as in the symmetric case. Vertical collusion implying ex post efficient decision rules, i.e., $\varepsilon=0$, its analysis is as in the asymmetric case with ex post efficient rules (Sections 6.3 and 6.4).

\section{Conclusion}

We have characterized the optimal incentive contracts for experts and the optimal organization of delegated expertise in different collusion environments. With a single expert, adverse selection restricts the set of feasible contracts, leading to returns to scale due to agency costs. Absent collusion, a two-expert organization is optimal. Indeed, it relaxes some adverse selection constraints, which increases the set of feasible contracts, leading to lower agency costs. However, this organization is prone to horizontal and vertical collusion, which reduce the set of feasible contracts. When both are possible, a one-expert organization is optimal.

In our model, different sets of feasible contracts correspond to different possibilities of collusion. As we have emphasized, these various nexi of collusion themselves can arise from limits in the control of communication between experts and between experts and the principal. An alternative interpretation of the different sets of feasible contracts is that they correspond to different departures from complete contracting. Our analysis shows that optimal collusion-proof 
contracts are relatively simple so that they can be interpreted as being incomplete.

Our analysis has abstracted from a number of technical and conceptual issues.

We have assumed that the signals were acquired simultaneously. This fits situations in which the principal cannot control the timing of reports or time constraints make sequential learning impossible. This timing allows for a simpler presentation of the interaction between the expert's two tasks. An earlier draft studied sequential information gathering (Gromb and Martimort [14]). The main difference with the simultaneous case is that, given our focus on ex post efficient decision rules, the second signal is collected only if the first report is positive. In particular, this implies that the agency cost for the second signal equals that of the simultaneous case but weighted by the probability that the first signal is positive. These differences notwithstanding, contracting issues are similar and, while the optimal contracts are different, the main results of the simultaneous case carry over to the sequential case.

We have assumed experts to be unbiased and to respond only to monetary incentives. Biases are an important concern for experts. In Political Science for instance, lobbyists are often viewed as biased experts eager to influence decision-makers (Laux [29], Dur and Swank [11]). Biased experts can be modeled by introducing non-transferable private benefits contingent on the outcome (Baliga and Sjöstrom [2]). These benefits would provide part of the expert's incentives to gather information. If the expert is a priori biased in favor of the project, inducing him to gather positive (resp. negative) signals is easier (resp. harder). These biases would affect the incentive problems but not our qualitative results.

We have assumed the expert's degree of expertise to be set and known. Situations in which the expert is privately informed about his skill (Osband [36]) or learns about it with experience (Dequiedt, Gromb and Martimort [8]) constitute interesting avenues of research.

In our model, a contracting externality arises between two signal-gathering tasks. One could study externalities arising between information-gathering and other tasks. For instance, should the planning of a project and its implementation be separated or centralized (Hirao [17], Lewis and Sappington [30], Laux [29], Khalil, Kim and Shin [23], Jeon [22])? Our approach, and particularly our study of collusion, could be applied to these questions.

\section{References}

[1] S. Baiman, J. Demski, Economically optimal performance evaluation and control systems, J. of Accounting Research 18 (1980), 189-220.

[2] S. Baliga, T. Sjöstrom, Optimal design of peer review and self-assessment schemes, RAND J. Econ. 32 (2001), 27-51.

[3] D. Baron, D. Besanko, Information, control and organizational structure, J. of Economics 
and Management Strategy 1 (1992), 237-275.

[4] D. Baron, D. Besanko, Informational alliance, Rev. Econ. Stud 66 (1999), 743-768.

[5] Y. Che, J. Kim, Robustly collusion-proof implementation, Econometrica 74 (2006), 10631107.

[6] J. Demski, D. Sappington, Optimal incentive contracts with multiple agents, J. Econ. Theory 33 (1984), 152-171.

[7] J. Demski, D. Sappington, Delegated expertise, Journal of Accounting Research 25 (1987), 68-89.

[8] V. Dequiedt, D. Gromb, D. Martimort, The dynamics of delegated expertise, mimeo IDEI Toulouse and London Business School, 2004.

[9] V. Dequiedt, D. Martimort, Delegated monitoring versus arm's length contracting, International Journal of Industrial Organization 22 (2004), 951-981.

[10] M. Dewatripont, J. Tirole, Advocates, J. Polit. Economy 107 (1999), 1-39.

[11] R. Dur, O. Swank, Producing and manipulating information, Economic Journal 115 (2005), 185-199.

[12] D. Gerardi, L. Yariv, "Information acquisition in committees, mimeo Yale University, 2006.

[13] R. Gilbert, M. Riordan, Regulating complementary products: a comparative institutional analysis, RAND J. Econ. 26 (1995), 243-256.

[14] D. Gromb, D. Martimort, The organization of delegated expertise, mimeo IDEI Toulouse and London Business School, 2003.

[15] J. Harrison, Reconceptualizing the expert witness: social costs, current controls and proposed responses, Yale Journal of Regulation 18 (2001), 252-314.

[16] M.A. Hermitte, L'expertise scientifique à finalité politique, réflexions sur l'organisation et la responsabilité des experts, Justices 8 (1997), 79-103.

[17] Y. Hirao, Task assignment and agency structures, Journal of Economics and Management Strategy 2 (1994), 299-323.

[18] B. Holmström, Moral hazard and observability, Bell Journal of Economics, 10 (1979), 74-91.

[19] B. Holmström, P. Milgrom, Regulating trade among agents, Journal of Institutional and Theoretical Economics 146 (1990), 85-105.

[20] B. Holmström, P. Milgrom, Multitask principal-agent analyses: incentive contracts, asset ownership and job design, J. Law, Econ., Organ. 7 (1991), 24-52.

[21] H. Itoh, Cooperation in hierarchical organizations: an incentive perspective, J. Law, Econ., 
Organ. 60 (1994), 410-427.

[22] D.S. Jeon, A theory of informational flows, mimeo University Pompeu Fabra, 2003.

[23] F. Khalil, D. Kim, D. Shin, Optimal task design: to integrate or separate planning and implementation? Journal of Economics and Management Strategy 14 (2006), 269-291.

[24] J.J. Laffont, D. Martimort, Collusion and delegation, RAND J. Econ. 29 (1998), 280-305.

[25] J.J. Laffont, D. Martimort, Separation of powers against collusive behavior, RAND J. Econ. 30 (1999), 232-263.

[26] J.J. Laffont, D. Martimort, Mechanism design with collusion and correlation, Econometrica 68 (2000), 309-342.

[27] R. Lambert, Executive effort and selection of risky projects, RAND J. Econ. 17 (1986), $77-88$

[28] C. Laux, Limited liability and incentive contracting with multiple projects, RAND J. Econ. 32 (2001a) 514-526.

[29] C. Laux, Delegated information acquisition and capital budgeting: on the separation of project evaluation and project management, Journal of Institutional and Theoretical Economics 157 (2001b), 591-607.

[30] T. Lewis, D. Sappington, Information management in incentive problems, J. Polit. Economy 105 (1997), 796-820.

[31] A. Ma, Unique implementation of incentive contracts with many agents, Rev. Econ. Stud 55 (1988), 555-572.

[32] I. Macho-Stadler, D. Perez-Castrillo, Moral hazard with several agents: the gains from cooperation, International Journal of Industrial Organization 11 (1993), 73-100.

[33] J. Malcomson, Principal and expert agent, mimeo Oxford University, 2001.

[34] D. Mookherjee, Optimal incentive schemes with many agents, Rev. Econ. Stud 51 (1984), 433-446.

[35] D. Mookherjee, M. Tsumagari, The organization of supplier networks: effects of delegation and intermediation, Econometrica 72 (2004), 1179-1219.

[36] K. Osband, Optimal forecasting incentives, J. Polit. Economy 97 (1989), 1091-1112.

[37] R. Ramakrishan, A. Thakor, Cooperation versus competition in agency, J. Law, Econ., Organ. 7 (1991), 248-283.

[38] S. Severinov, The value of information and optimal organization, mimeo Duke University, 2005. 
[39] J. Tirole, Hierarchies and bureaucracies, J. Law, Econ., Organ. 2 (1986), 181-214.

\section{Appendix}

Proof of Lemma 1: We do not impose $\underline{t}=0$. The principal's problem is then:

$$
\begin{gathered}
\min _{\left\{\bar{t}, \underline{t}, t_{0}\right\}} p(\bar{\sigma})(\nu(\bar{\sigma}) \bar{t}+(1-\nu(\bar{\sigma})) \underline{t})+p(\underline{\sigma}) t_{0} \\
\text { subject to } \\
\nu(\bar{\sigma}) \bar{t}+(1-\nu(\bar{\sigma})) \underline{t} \geq t_{0}+\frac{\psi}{p(\bar{\sigma})} \\
t_{0} \geq \nu(\underline{\sigma}) \bar{t}+(1-\nu(\underline{\sigma})) \underline{t}+\frac{\psi}{p(\underline{\sigma})} \\
\underline{t} \geq 0 .
\end{gathered}
$$

The expert's participation and limited liability constraints

$$
p(\bar{\sigma}) \nu(\bar{\sigma}) \bar{t}+p(\underline{\sigma}) t_{0}-\psi \geq 0, \text { and } \bar{t}, t_{0} \geq 0
$$

are easily shown to be satisfied, and omitted henceforth. Since $\nu(\bar{\sigma})>\nu(\underline{\sigma})$, one can keep the expected transfer constant and (39) unchanged, and relax (40) by reducing $\underline{t}$. Hence (41) is binding. The remaining constraints, (39) and (40), define a cone in the positive quadrant of the $\left(t_{0}, \bar{t}\right)$ space, and the optimum is reached at its origin, i.e., (39) and (40) are binding.

Proof of Lemma 3: (15) can be written as:

$$
(1-p(\bar{\sigma}, \bar{\sigma})-p(\underline{\sigma})) t_{0} \geq(p(\bar{\sigma}) \nu(\bar{\sigma})-p(\bar{\sigma}, \bar{\sigma}) \nu(\bar{\sigma}, \bar{\sigma})) \bar{t}+\psi,
$$

which can be written as

$$
p(\bar{\sigma}, \underline{\sigma}) t_{0} \geq p(\bar{\sigma}, \underline{\sigma}) \nu(\bar{\sigma}, \underline{\sigma}) \bar{t}+\psi
$$

which, given that $\nu(\bar{\sigma}, \underline{\sigma})=\nu$, can be written as:

$$
t_{0} \geq \nu \bar{t}+\frac{\psi}{p(\bar{\sigma}, \underline{\sigma})}
$$

which implies (12) (and, incidentally, (13)). Since (45) implies that $t_{0}>\nu \bar{t}$, (14) boils down to:

$$
p(\bar{\sigma}, \bar{\sigma}) \nu(\bar{\sigma}, \bar{\sigma}) \bar{t}+(1-p(\bar{\sigma}, \bar{\sigma})) t_{0}-2 \psi \geq t_{0}
$$

which following similar calculations, can be written as:

$$
\nu(\bar{\sigma}, \bar{\sigma}) \bar{t} \geq t_{0}+2 \frac{\psi}{p(\bar{\sigma}, \bar{\sigma})} .
$$

Hence (45) and (47) define a cone in the positive quadrant of the $\left(t_{0}, \bar{t}\right)$ space, and the optimum is reached at its extreme point. 
Proof of Lemma 4: The moral hazard incentive constraint can be rewritten as:

$$
p(\bar{\sigma} \mid \bar{\sigma}) \nu(\bar{\sigma}, \bar{\sigma}) \bar{t}+p(\underline{\sigma} \mid \bar{\sigma}) t_{G} \geq p(\bar{\sigma} \mid \bar{\sigma}) t_{B}+p(\underline{\sigma} \mid \bar{\sigma}) t_{0}+\frac{\psi}{p(\bar{\sigma})},
$$

and

$$
p(\bar{\sigma} \mid \underline{\sigma}) t_{B}+p(\underline{\sigma} \mid \underline{\sigma}) t_{0} \geq p(\bar{\sigma} \mid \underline{\sigma}) \nu(\bar{\sigma}, \underline{\sigma}) \bar{t}+p(\underline{\sigma} \mid \underline{\sigma}) t_{G}+\frac{\psi}{p(\underline{\sigma})} .
$$

Consider first the following problem: For a given value $T_{G}$ of $p(\bar{\sigma} \mid \bar{\sigma}) \nu(\bar{\sigma}, \bar{\sigma}) \bar{t}+p(\underline{\sigma} \mid \bar{\sigma}) t_{G}$, keeping both the LHS of (48) and the expected transfer unchanged, what is the value of $t_{G}$ minimizing the RHS of (49)? We solve:

$$
\begin{gathered}
\min _{\left\{\bar{t}, t_{G}\right\}} p(\bar{\sigma} \mid \underline{\sigma}) \nu(\bar{\sigma}, \underline{\sigma}) \bar{t}+p(\underline{\sigma} \mid \underline{\sigma}) t_{G} \\
\text { subject to } \\
T_{G}=p(\bar{\sigma} \mid \bar{\sigma}) \nu(\bar{\sigma}, \bar{\sigma}) \bar{t}+p(\underline{\sigma} \mid \bar{\sigma}) t_{G} \\
t_{G} \geq 0 .
\end{gathered}
$$

This problem can be rewritten as:

$$
\min _{\left\{t_{G}\right\}} \frac{p(\bar{\sigma} \mid \underline{\sigma}) \nu(\bar{\sigma}, \underline{\sigma})}{p(\bar{\sigma} \mid \bar{\sigma}) \nu(\bar{\sigma}, \bar{\sigma})}\left(T_{G}-p(\underline{\sigma} \mid \bar{\sigma}) t_{G}\right)+p(\underline{\sigma} \mid \underline{\sigma}) t_{G} \quad \text { subject to }(51) .
$$

The coefficient of $t_{G}$ in this problem is $p(\underline{\sigma} \mid \underline{\sigma})-\frac{p(\bar{\sigma} \mid \underline{\sigma}) \nu(\bar{\sigma}, \underline{\sigma})}{p(\bar{\sigma} \mid \bar{\sigma}) \nu(\bar{\sigma}, \bar{\sigma})} p(\underline{\sigma} \mid \bar{\sigma})=\frac{(2 \theta-1) \nu}{p(\underline{\sigma})}>0$ and thus the minimum is obtained when (51) is binding. Similarly, consider the following problem: For a given value $T_{B}$ of $p(\bar{\sigma} \mid \underline{\sigma}) t_{B}+p(\underline{\sigma} \mid \underline{\sigma}) t_{0}$ keeping both the LHS of (49) and the expected transfer unchanged, what is the value of $t_{B}$ minimizing the RHS of (48)? We solve:

$$
\begin{array}{cc}
\min _{\left\{t_{B}, t_{0}\right\}} & p(\bar{\sigma} \mid \bar{\sigma}) t_{B}+p(\underline{\sigma} \mid \bar{\sigma}) t_{0} \\
& \text { subject to } \\
T_{B}=p(\bar{\sigma} \mid \underline{\sigma}) t_{B}+p(\underline{\sigma} \mid \underline{\sigma}) t_{0} \\
t_{B} \geq 0 .
\end{array}
$$

This problem can be rewritten as:

$$
\min _{\left\{t_{B}\right\}} p(\bar{\sigma} \mid \bar{\sigma}) t_{B}+\frac{p(\underline{\sigma} \mid \bar{\sigma})}{p(\underline{\sigma} \mid \underline{\sigma})}\left(T_{B}-p(\bar{\sigma} \mid \underline{\sigma}) t_{B}\right) \quad \text { subject to (53). }
$$

The coefficient of $t_{B}$ in this problem is $p(\bar{\sigma} \mid \bar{\sigma})-\frac{p(\underline{\sigma} \mid \bar{\sigma})}{p(\underline{\sigma} \underline{\sigma})} p(\bar{\sigma} \mid \underline{\sigma})=\frac{(2 \theta-1)^{2} \nu(1-\nu)}{p(\bar{\sigma}) p(\underline{\sigma}, \underline{\sigma})}>0$ and thus the minimum is obtained when (53) is binding. With this in mind, the principal's problem can be rewritten as:

$$
\begin{gathered}
\min _{\left\{\bar{t}, t_{0}\right\}} p(\bar{\sigma}, \bar{\sigma}) \nu(\bar{\sigma}, \bar{\sigma}) \bar{t}+p(\underline{\sigma}, \underline{\sigma}) t_{0} \\
\text { subject to } \\
p(\bar{\sigma} \mid \bar{\sigma}) \nu(\bar{\sigma}, \bar{\sigma}) \bar{t} \geq p(\underline{\sigma} \mid \bar{\sigma}) t_{0}+\frac{\psi}{p(\bar{\sigma})} \quad \text { and } \quad p(\underline{\sigma} \mid \underline{\sigma}) t_{0} \geq p(\bar{\sigma} \mid \underline{\sigma}) \nu(\bar{\sigma}, \underline{\sigma}) \bar{t}+\frac{\psi}{p(\underline{\sigma})} .
\end{gathered}
$$


These two constraints define a cone in the positive quadrant of the $\left(t_{0}, \bar{t}\right)$ space and the optimum is reached at its extreme point. Simple manipulations complete the proof.

Proof of Proposition 3: First, note that the collusive equilibria arising in the optimal contract absent collusion are strict, i.e., cannot be eliminated by a small deviation from the optimal contract. Second, since the experts do not observe each other's effort, standard implementation techniques used for moral hazard contexts (e.g. Ma [31]) cannot be used to eliminate unwanted equilibria. However, we use a similar approach, detailed below. Third, outcomes in which only one expert is informed are not an issue: The only reason the expert would gather information would be to report it, but in that case, the other expert's moral hazard constraint ensures that he does the same. Hence, in potential unwanted pure strategy equilibria, the experts remain uninformed and report $(\underline{\sigma}, \underline{\sigma}),(\bar{\sigma}, \bar{\sigma}),(\bar{\sigma}, \underline{\sigma})$ or $(\underline{\sigma}, \bar{\sigma})$.

Since the two experts can receive different contracts, we index the transfers by $A$ or $B$. We rule out unwanted pure strategy equilibria by ensuring that at least one expert strictly prefers deviating. Given such a putative equilibrium, two deviations are possible: Either an expert prefers to remain uninformed but make a different report or an expert prefers to collect and report a signal. Hence, a contract implements uniquely the truthful and obedient outcome:

$$
\begin{aligned}
\max \left\{t_{G}^{A}, p(\bar{\sigma}) t_{G}^{A}+p(\underline{\sigma}) t_{0}^{A}-\psi\right\}>t_{0}^{A} \text { or } \max \left\{t_{G}^{B}, p(\bar{\sigma}) t_{G}^{B}+p(\underline{\sigma}) t_{0}^{B}-\psi\right\}>t_{0}^{B} \\
\max \left\{t_{B}^{A}, p(\bar{\sigma}) \nu(\bar{\sigma}) \bar{t}^{A}+p(\underline{\sigma}) t_{B}^{A}-\psi\right\}>\nu \bar{t}^{A} \text { or } \max \left\{t_{B}^{B}, p(\bar{\sigma}) \nu(\bar{\sigma}) \bar{t}^{B}+p(\underline{\sigma}) t_{B}^{B}-\psi\right\}>\nu \bar{t}^{B} \\
\max \left\{t_{0}^{A}, p(\bar{\sigma}) t_{G}^{A}+p(\underline{\sigma}) t_{0}^{A}-\psi\right\}>t_{G}^{A} \text { or } \max \left\{\nu \bar{t}^{B}, p(\bar{\sigma}) \nu(\bar{\sigma}) \bar{t}^{B}+p(\underline{\sigma}) t_{B}^{B}-\psi\right\}>t_{B}^{B} \\
\max \left\{t_{0}^{B}, p(\bar{\sigma}) t_{G}^{B}+p(\underline{\sigma}) t_{0}^{B}-\psi\right\}>t_{G}^{B} \text { or } \max \left\{\nu \bar{t}^{A}, p(\bar{\sigma}) \nu(\bar{\sigma}) \bar{t}^{A}+p(\underline{\sigma}) t_{B}^{A}-\psi\right\}>t_{B}^{A} .
\end{aligned}
$$

For example, (55) states that at least one expert must prefer deviating from $(\underline{\sigma}, \underline{\sigma})$. Expert $i \in\{A, B\}$ could deviate by remaining uninformed and reporting $\bar{\sigma}$. In that case, his payoff would be $t_{G}^{i}$. Alternatively, he could deviate by acquiring and reporting a signal, in which case his payoff would be $p(\bar{\sigma}) t_{G}^{i}+p(\underline{\sigma}) t_{0}^{i}-\psi$. The other constraints correspond to deviations from $(\bar{\sigma}, \bar{\sigma})$ or $(\bar{\sigma}, \underline{\sigma})$, and $(\underline{\sigma}, \bar{\sigma})$ respectively. Note that the same expert's contract cannot satisfy both the inequalities in (55) and (57) or both those in (56) and (58). Therefore, the optimal scheme must be asymmetric.

Denote $\underline{t}^{i *}, t_{G}^{i *}, t_{B}^{i *}, \bar{t}^{i *}$ and $t_{0}^{i *}$ the optimal transfers for expert $i \in\{A, B\}$. The inequalities in (55)-(58) being strict, the feasible contract set is open and an optimal contract might not be properly defined. We will nevertheless abuse notation and call optimal the solution of the principal's problem when strict inequalities are replaced by weak ones with the proviso that indifferences can be broken with infinitesimal changes in the transfers. We write $t=x^{+}$to mean that $t$ is larger and arbitrarily close to $x$.

Note that $p(\bar{\sigma}) t_{G}^{i}+p(\underline{\sigma}) t_{0}^{i}-\psi>t_{0}^{i}$ implies $t_{G}^{i}>t_{0}^{i}$. Hence (55) can be rewritten as $t_{G}^{i}>t_{0}^{i}$. Now, consider the restricted problem:

$$
\min _{\left(\bar{t}^{A}, t_{0}^{A}, t_{G}^{A}, t_{B}^{A}\right)} p(\bar{\sigma}, \bar{\sigma}) \nu(\bar{\sigma}, \bar{\sigma}) \bar{t}^{A}+p(\bar{\sigma}, \underline{\sigma})\left(t_{G}^{A}+t_{B}^{A}\right)+p(\underline{\sigma}, \underline{\sigma}) t_{0}^{A}
$$


subject to the limited liability constraints, $A$ 's moral hazard incentive constraint (20) and the deviating condition (55) that will now be taken as a weak inequality: $t_{G}^{A} \geq t_{0}^{A}$. Absent (55), the solution would be the no-collusion optimal contract, which violates (55). Hence, (55) is binding: $t_{G}^{A}=\left(t_{0}^{A}\right)^{+}$. Inserting $t_{G}^{A}=t_{0}^{A}$ into (20) yields:

$$
p(\bar{\sigma}, \bar{\sigma}) \nu(\bar{\sigma}, \bar{\sigma}) \bar{t}^{A}+p(\bar{\sigma}, \underline{\sigma}) t_{B}^{A}-\psi \geq \max \left\{p(\bar{\sigma}) \nu(\bar{\sigma}) \bar{t}^{A}, p(\bar{\sigma}) t_{B}^{A}\right\} .
$$

This constraint does not depend on $t_{0}^{A}$, which is set at zero to minimize the objective (i.e., the limited liability constraint $t_{0} \geq 0$ is binding). (20) is binding and can be rewritten as:

$$
t_{B}^{A}=\frac{\psi}{p(\bar{\sigma}, \underline{\sigma})}+\nu \bar{t}^{A} \quad \text { and } \quad \nu(\bar{\sigma}, \bar{\sigma}) \bar{t}^{A}=\frac{\psi}{p(\bar{\sigma}, \bar{\sigma})}+t_{B}^{A} .
$$

Hence we have:

$$
t_{G}^{A *}=0^{+}, \quad t_{0}^{A *}=0 \quad \text { and } \quad t_{B}^{A *}=\nu(\bar{\sigma}) \bar{t}^{A *}=\frac{\psi}{(1-\nu)(2 \theta-1)(1-\theta)} .
$$

A few remarks complete the proof. First, $t_{B}^{A}>\nu \bar{t}^{A}$ so that A's contract satisfies the inequality in (56). Second, the same expert cannot satisfy both the inequalities in (55) and (57) or both those in (56) and (58). Hence, the optimal scheme must have expert $A$ 's contract satisfy the inequality in (55) and (56), and expert $B$ 's satisfy those in (57) and (58). Third, this implies that $A$ 's contract is the solution to the restricted problem above, while $B$ 's contract must be the optimal contract absent collusion as it satisfies the inequalities in (57) and (58). Finally, $U^{A *}$ obtains from (20) being binding.

Proof of Proposition 4: Given (25), the principal's problem is:

$$
\min _{\left(\bar{t}, t_{0}, t_{G}, t_{B}\right)} p(\bar{\sigma}, \bar{\sigma}) \nu(\bar{\sigma}, \bar{\sigma}) \bar{t}+(1-p(\bar{\sigma}, \bar{\sigma})) t_{0}
$$

subject to (25), (26), (27) and each expert's moral hazard incentive constraint (20), which can be written as:

$$
p(\bar{\sigma}, \bar{\sigma}) \nu(\bar{\sigma}, \bar{\sigma}) \bar{t}+(1-p(\bar{\sigma}, \bar{\sigma})) t_{0}-\psi \geq p(\bar{\sigma}) \nu(\bar{\sigma}) \bar{t}+p(\underline{\sigma}) t_{G}
$$

and

$$
p(\bar{\sigma}, \bar{\sigma}) \nu(\bar{\sigma}, \bar{\sigma}) \bar{t}+(1-p(\bar{\sigma}, \bar{\sigma})) t_{0}-\psi \geq p(\bar{\sigma})\left(2 t_{0}-t_{G}\right)+p(\underline{\sigma}) t_{0} .
$$

First, (60) can be rewritten as:

$$
(1-p(\bar{\sigma}, \bar{\sigma})-2 p(\underline{\sigma})) t_{0}-p(\underline{\sigma})\left(2 t_{0}-t_{G}\right) \geq \psi+(p(\bar{\sigma}) \nu(\bar{\sigma})-p(\bar{\sigma}, \bar{\sigma}) \nu(\bar{\sigma}, \bar{\sigma})) \bar{t},
$$

which is violated for $t_{G}=2 t_{0}$ because $(1-p(\bar{\sigma}, \bar{\sigma}))-2 p(\underline{\sigma})=p(\underline{\sigma}, \bar{\sigma})-p(\underline{\sigma})<0$. Hence, the constraint $t_{G} \leq 2 t_{0}$ (i.e., $t_{B} \geq 0$ ) is slack. For now, we ignore the constraint $t_{G} \geq 0$ and check later that it holds. Second, (60) is the only remaining upper bound for $t_{G}$. Since $t_{G}$ is not in the principal's objective, (60) is binding. Replacing $t_{G}$ in (61) yields:

$$
\nu \theta(\theta-p(\bar{\sigma})) \bar{t} \geq \psi+\left(p(\bar{\sigma}, \bar{\sigma})-p(\bar{\sigma})^{2}\right) t_{0} .
$$


Third, as the only lower bound for $t_{0},(27)$ must be binding, i.e., $t_{0}=\nu \bar{t}$. This implies that $(26)$ is slack. Next, as the only lower bound for $\bar{t},(61)$ must be binding. This yields the transfers in the Lemma. Finally, we check that $t_{G} \geq 0$. From (60), we have:

$$
\begin{aligned}
p(\underline{\sigma}) t_{G} & =-(p(\bar{\sigma}) \nu(\bar{\sigma})-p(\bar{\sigma}, \bar{\sigma}) \nu(\bar{\sigma}, \bar{\sigma})) \bar{t}+(1-p(\bar{\sigma}, \bar{\sigma})) t_{0}-\psi \\
& =\frac{[\theta(1-\theta)+(1-(1-\nu)(2 \theta-1)) p(\underline{\sigma})] \psi}{(1-\nu)(2 \theta-1) p(\underline{\sigma})}>0
\end{aligned}
$$

We can derive each expert's rent as (61)'s LHS. Hence denoting $\underline{t}^{2,2, h}, t_{G}^{2,2, h}, t_{B}^{2,2, h}, \bar{t}^{2,2, h}, t_{0}^{2,2, h}$ and $U^{2,2, h}$ the optimal transfers and rent, we have the optimal transfers:

$$
\begin{gathered}
\nu \vec{t}^{2,2, h}=t_{0}^{2,2, h}=\frac{\psi}{(1-\nu)(2 \theta-1) p(\underline{\sigma})}, \\
t_{G}^{2,2, h}=\frac{(1-(1-\nu)(2 \theta-1) p(\bar{\sigma})) \psi}{(1-\nu)(2 \theta-1) p(\underline{\sigma})} \quad \text { and } \quad t_{B}^{2,2, h}=\frac{(1+(1-\nu)(2 \theta-1) p(\bar{\sigma})) \psi}{(1-\nu)(2 \theta-1) p(\underline{\sigma})} .
\end{gathered}
$$

and the corresponding rent for each expert:

$$
U^{2,2, h}=\frac{(1+(1-\nu)(2 \theta-1) p(\bar{\sigma})) \psi}{(1-\nu)(2 \theta-1) p(\underline{\sigma})} .
$$

Note that $t_{B}^{2,2, h}>t_{0}^{2,2, h}>t_{G}^{2,2, h}>0$. The optimal contract remains report-based but the principal can no longer fully punish conflicting reports because $2 t_{0}=t_{G}+t_{B}$ must hold. The intuition for this ranking is as follows. On the one hand, $t_{G}$ and $t_{B}$ must be somewhat even. Minimizing $t_{G}$ (setting $t_{G}=0$ ) would mean maximizing $t_{B}$ (setting $t_{B}=2 t_{0}$ ). The expert would be tempted not to gather information and always recommend rejecting the project. Correcting this bias would require increasing $\bar{t}$. Similarly, minimizing $t_{B}$ would bias the expert in favor of the project and require increasing $t_{0}$. On the other hand, other things being equal, increasing $\bar{t}$ is less costly than increasing $t_{0}$ because the former transfer is contingent on a more informative outcome. Therefore, it is optimal to depart from $t_{G}=t_{B}$ and increase $t_{B}$.

The two-expert organization is optimal if and only if $U^{1,2} \geq 2 U^{2,2, h}$.

$$
U^{1,2}-2 U^{2,2, h}=\frac{N(\nu, \theta)}{(1-\nu)(2 \theta-1)(1-\theta) p(\underline{\sigma})} \psi
$$

where the numerator is:

$$
N(\nu, \theta) \equiv 2(2 \theta-1)^{2}(1-\theta) \nu^{2}-(2 \theta-1)\left(-6 \theta^{2}+9 \theta-2\right) \nu-\theta\left(4 \theta^{2}-9 \theta+4\right) .
$$

For all $\theta, N(\nu, \theta)<0$ if and only if $\nu \in\left(\nu_{1}(\theta), \nu_{2}(\theta)\right)$ where:

$$
\begin{aligned}
& \nu_{1}(\theta)=\frac{-6 \theta^{2}+9 \theta-2-\sqrt{4\left(1-\theta^{3}\right)(1-\theta)+\theta^{2}}}{4(2 \theta-1)(1-\theta)} \\
& \nu_{2}(\theta)=\frac{-6 \theta^{2}+9 \theta-2+\sqrt{4\left(1-\theta^{3}\right)(1-\theta)+\theta^{2}}}{4(2 \theta-1)(1-\theta)}
\end{aligned}
$$


We need to determine whether and when $\nu_{1}(\theta)$ and $\nu_{2}(\theta)$ are within $(0,1)$. For all $\theta \in[1 / 2,1]$, $N(1, \theta)=-\theta(1-\theta) \leq 0$, which implies $\nu_{2}(\theta) \geq 1$. Hence, for all $\theta \in[1 / 2,1], N(\nu, \theta)<0$ if and only if $\nu<\nu^{*}(\theta) \equiv \max \left\{0, \nu_{1}(\theta)\right\} \in[0,1]$.

Now, notice that for all $\nu \in(0,1), N(\nu, 1 / 2)=-1 / 4<0$ and $N(\nu, 1)=1-\nu>0$. Showing that $\frac{d \nu_{1}(\theta)}{d \theta}>0$ would complete the proof. Indeed, this would imply $\exists \theta_{0} \in(1 / 2,1)$ such that $\nu^{*}(\theta)$ is a strictly increasing one-to-one mapping between $\left[\theta_{0}, 1\right]$ and $[0,1]$ so that $\theta^{*} \equiv\left(\nu^{*}\right)^{-1}$ would be as stated in the proposition.

We now prove that $\frac{d \nu_{1}(\theta)}{d \theta}>0$ over $[1 / 2,1]$. Define the following functions over $[1 / 2,1]$ :

$$
f(\theta) \equiv 10-21 \theta+2 \theta^{2}\left(3+6 \theta-4 \theta^{2}\right) \quad \text { and } \quad g(\theta) \equiv(4 \theta-3) \sqrt{4(1-\theta)+(2 \theta-1)^{2} \theta^{2}} .
$$

We have:

$$
\frac{d \nu_{1}(\theta)}{d \theta}=\frac{f(\theta)+g(\theta)}{4(2 \theta-1)^{2}(1-\theta)^{2} \sqrt{4(1-\theta)+(2 \theta-1) \theta^{2}}} .
$$

To show that $f(\theta)+g(\theta)>0$, we study the sign of $f(\theta)$ and $g(\theta)$ over $[1 / 2,1]$. Clearly, $g(\theta) \geq 0$ if and only if $\theta \in[3 / 4,1]$. We have:

$$
f^{\prime}(\theta)=-21+12 \theta+36 \theta^{2}-32 \theta^{3} \quad \text { and } \quad f^{\prime \prime}(\theta)=12\left(1+6 \theta-8 \theta^{2}\right) .
$$

Since $f^{\prime \prime}(\theta)=0$ for $\theta_{1}=\frac{3-\sqrt{17}}{8}<0$ and $\theta_{2}=\frac{3+\sqrt{17}}{8} \in(3 / 4,1)$, we have $\max _{\theta \in[1 / 2,1]} f^{\prime}(\theta)=$ $f^{\prime}\left(\theta_{2}\right)=-4.4<0$. Hence $f^{\prime}<0$ over $[1 / 2,1]$. Note that $f(0.77)>0$ and $f(1)<0$.

Case 1: $\theta \in[1 / 2,3 / 4]$. Since $f(\theta)>0, f(\theta)^{2}>g(\theta)^{2}$ implies $\frac{d \nu_{1}(\theta)}{d \theta}>0$. We have:

$$
f(\theta)^{2}-g(\theta)^{2}=8(1-\theta)^{2}\left(8-20 \theta+\theta^{2}+36 \theta^{3}-26 \theta^{4}-8 \theta^{5}+8 \theta^{6}\right) .
$$

Hence, we only need to show that $h(\theta) \equiv \frac{f(\theta)^{2}-g(\theta)^{2}}{8(1-\theta)^{2}}>0$ for all $\theta \in[1 / 2,3 / 4]$. We have:

$$
h^{\prime}(\theta)=-2\left(10-\theta-54 \theta^{2}+52 \theta^{3}+20 \theta^{4}-24 \theta^{5}\right) .
$$

Since $\min _{\theta \in[1 / 2,3 / 4]}\left(-54 \theta^{2}+52 \theta^{3}\right)=\left(-54\left(\frac{9}{13}\right)^{2}+52\left(\frac{9}{13}\right)^{3}\right)<8.7$, we have for all $\theta \in[1 / 2,3 / 4]$,

$$
h^{\prime}(\theta)<-2\left[(10-8.7-\theta)+24 \theta^{4}\left(\frac{5}{6}-\theta\right)\right]<0 .
$$

Hence, $\min _{\theta \in[1 / 2,3 / 4]} h(\theta)=h(3 / 4)>0$.

Case 2: $\theta \in[3 / 4,0.77]$. Both $f(\theta)>0$ and $g(\theta)>0$, implying $\frac{d \nu_{1}(\theta)}{d \theta}>0$.

Case 3: $\theta \in[0.77,1]$. A sufficient condition for $\frac{d \nu_{1}(\theta)}{d \theta}>0$ is $h<0$. We have:

$$
\begin{gathered}
h^{\prime \prime}(\theta)=-2\left(-1-108 \theta+156 \theta^{2}+80 \theta^{3}-120 \theta^{4}\right) \\
h^{\prime \prime \prime}(\theta)=-8\left(-27+78 \theta+60 \theta^{2}-120 \theta^{3}\right) \quad \text { and } \quad h^{\prime \prime \prime \prime}(\theta)=-16\left(39+60 \theta-180 \theta^{2}\right)
\end{gathered}
$$

$\arg \min _{\theta \in[0,1]} h^{\prime \prime \prime \prime}(\theta)=1 / 6<0.77$. Hence $\min _{\theta \in[0.77,1]} h^{\prime \prime \prime \prime}(\theta)=h^{\prime \prime \prime \prime}(0.77)=344>0$. Hence $h^{\prime \prime}$ is convex over $[0.77,1]$ and $\max _{\theta \in[0.77,1]} h^{\prime \prime}(\theta)=\max \left\{h^{\prime \prime}(0.77), h^{\prime \prime}(1)\right\}=\max \{-5.3,-14\}<0$. 
Hence $\max _{\theta \in[0.77,1]} h^{\prime}(\theta)=h^{\prime}(0.77)=-2.97<0$. Hence $\max _{\theta \in[0.77,1]} h(\theta)=h(0.77)=-0.01<$ 0 .

Proof of Proposition 5: The optimal transfers $\underline{t}^{2,2, v}, t_{G}^{2,2, v}, t_{B}^{2,2, v}, \vec{t}^{2,2, v}$ and $t_{0}^{2,2, v}$ solve:

$\min _{\left\{\bar{t}, t_{G}, t_{B}, t_{0}\right\}} p(\bar{\sigma}, \bar{\sigma}) \nu(\bar{\sigma}, \bar{\sigma}) \bar{t}+p(\bar{\sigma}, \underline{\sigma})\left(t_{G}+t_{B}\right)+p(\underline{\sigma}, \underline{\sigma}) t_{0} \quad$ subject to (18), (19), (20), (21) and (28).

Given $t_{B}=t_{0},(20)$ can be written as:

$$
p(\bar{\sigma} \mid \bar{\sigma}) \nu(\bar{\sigma} \mid \bar{\sigma}) \bar{t}+p(\underline{\sigma} \mid \bar{\sigma}) t_{G} \geq t_{0}+\frac{\psi}{p(\bar{\sigma})},
$$

and

$$
t_{0} \geq p(\underline{\sigma} \mid \bar{\sigma}) \nu(\bar{\sigma}, \underline{\sigma}) \bar{t}+p(\bar{\sigma} \mid \bar{\sigma}) t_{0}+\frac{\psi}{p(\bar{\sigma})} .
$$

(18) and (19) are obtained by eliminating the terms in $\psi$. Clearly, for a given expected value of $p(\bar{\sigma} \mid \bar{\sigma}) \nu(\bar{\sigma} \mid \bar{\sigma}) \bar{t}+p(\underline{\sigma} \mid \bar{\sigma}) t_{G}$, setting $t_{G}=0$ relaxes (79). From this, it is straightforward to show that the optimal collusion-proof contract implementing the first-best decision rule is such that :

$$
\vec{t}^{2,2, v}=\frac{\psi}{\nu(1-\nu)(2 \theta-1) \theta}, \quad t_{G}^{2,2, v}=0, \quad U^{2,2, v}=t_{0}^{2,2, v}=t_{B}^{2,2, v}=\frac{\psi}{(1-\nu)(2 \theta-1)}=U^{1,1}
$$

Proof of Proposition 6: Each expert's moral hazard constraint can now be written as:

$$
p(\bar{\sigma}, \bar{\sigma}) \nu(\bar{\sigma}, \bar{\sigma}) \bar{t}+(1-p(\bar{\sigma}, \bar{\sigma})) t_{0}-\psi \geq \max \left\{t_{0}, p(\bar{\sigma}) \nu(\bar{\sigma}) \bar{t}+p(\underline{\sigma}) t_{0}\right\}
$$

which is easily shown to imply constraint (26), and the adverse selection constraints. (80) can be written as two constraints defining a cone in the positive quadrant of the $\left(t_{0}, \bar{t}\right)$ space:

$$
\begin{gathered}
\nu(\bar{\sigma}, \bar{\sigma}) \bar{t} \geq t_{0}+\frac{\psi}{p(\bar{\sigma}, \bar{\sigma})}, \\
(1-p(\bar{\sigma}, \bar{\sigma})-p(\underline{\sigma})) t_{0} \geq(p(\bar{\sigma}) \nu(\bar{\sigma})-p(\bar{\sigma}, \bar{\sigma}) \nu(\bar{\sigma}, \bar{\sigma})) \bar{t}+\psi .
\end{gathered}
$$

The optimum is reached at the cone's origin.

Proof of Lemma 5: Denote $\underline{t} \equiv t(\bar{\sigma}, \underline{S}), \bar{\tau} \equiv \varepsilon \times t(\underline{\sigma}, \bar{S}), t_{0} \equiv(1-\varepsilon) \times t(\underline{\sigma}, \varnothing), \bar{T} \equiv$ $\nu(\bar{\sigma}) \bar{t}+(1-\nu(\bar{\sigma})) \underline{t}$ and $\underline{T} \equiv(\nu(\underline{\sigma}) \bar{\tau}+(1-\nu(\underline{\sigma})) \underline{\tau})+t_{0}$. The principal's problem is:

$$
\min _{\bar{T} \geq 0, \underline{T} \geq 0} p(\bar{\sigma}) \bar{T}+p(\underline{\sigma}) \underline{T}
$$

subject to adverse selection incentive constraints:

$$
\bar{T} \geq \underline{T}+(\nu(\bar{\sigma})-\nu(\underline{\sigma}))(\bar{\tau}-\underline{\tau}) \quad \text { and } \quad \underline{T} \geq \bar{T}-(\nu(\bar{\sigma})-\nu(\underline{\sigma}))(\bar{t}-\underline{t}),
$$

which are implied by the moral hazard incentive constraints:

$$
\begin{aligned}
& \bar{T} \geq \frac{\psi}{p(\bar{\sigma})}+\underline{T}+(\nu(\bar{\sigma})-\nu(\underline{\sigma}))(\bar{\tau}-\underline{\tau}), \\
& \underline{T} \geq \frac{\psi}{p(\underline{\sigma})}+\bar{T}-(\nu(\bar{\sigma})-\nu(\underline{\sigma}))(\bar{t}-\underline{t}) .
\end{aligned}
$$


Holding $\bar{T}$ fixed, (85) is relaxed when $\bar{t}$ is maximized and $\underline{t}$ minimized. Hence $\underline{t}=0$ is optimal. Holding $\underline{T}$ fixed, (84) is relaxed when $\underline{\tau}$ is maximized and $\bar{\tau}$ minimized. Hence $\bar{\tau}=0$ is optimal. Moreover, minimizing $t_{0}$ allows to increase $\underline{\tau}$, further relaxing (84). Hence $t_{0}=0$ is optimal.

With this in mind, (84) and (85) are binding and can be rewritten as:

$$
\begin{aligned}
p(\bar{\sigma}) \nu(\bar{\sigma}) \bar{t} & =\psi+p(\bar{\sigma})(1-\nu(\bar{\sigma})) \underline{\tau} \\
p(\underline{\sigma})(1-\nu(\underline{\sigma})) \underline{\tau} & =\psi+p(\underline{\sigma}) \nu(\underline{\sigma}) \bar{t}
\end{aligned}
$$

which yields the optimal transfers $\underline{\tau}^{1,1}$ and $\vec{t}^{1,1}$, and rent $U^{1,1}$ :

$$
U^{1,1}=\nu \bar{t}^{1,1}=(1-\nu) \underline{\tau}^{1,1}=\frac{\psi}{2 \theta-1} .
$$

Proof of Lemma 6: For brevity, we assume but do not prove that in the optimal contract, $t\left(\hat{\sigma}_{1}, \hat{\sigma}_{2}, \varnothing\right)=0$, because observing the outcome is more informative, and $t(\bar{\sigma}, \bar{\sigma}, \underline{S})=t(\underline{\sigma}, \underline{\sigma}, \bar{S})=$ 0 , because the expert should not be rewarded when the outcome contradicts both his reports.

Constraint (35)'s third term can be written as:

$$
\theta^{2} \nu \bar{t}+2 \theta(1-\theta)\left(\nu \tau_{1}+(1-\nu) \tau_{2}\right)+\theta^{2}(1-\nu) \underline{\tau}-2 \psi \geq \nu \tau_{1}+(1-\nu) \tau_{2},
$$

and constraint (36)'s first term can be written as:

$$
\theta^{2} \nu \bar{t}+2 \theta(1-\theta)\left(\nu \tau_{1}+(1-\nu) \tau_{2}\right)+\theta^{2}(1-\nu) \underline{\tau}-2 \psi \geq \theta \nu \bar{t}+\theta(1-\nu) \underline{\tau}-\psi
$$

With the notation $T \equiv \nu \bar{t}+(1-\nu) \underline{\tau}$ and $\hat{T} \equiv \nu \tau_{1}+(1-\nu) \tau_{2}$ and ignoring for now all constraints but (89) and (90), the principal's programme can be rewritten as:

$$
\left\{\begin{array}{c}
\min _{(T, \hat{T})} \theta^{2} T+2 \theta(1-\theta) \hat{T} \\
\theta^{2} T \geq 2 \psi+\left(1-2 \theta+2 \theta^{2}\right) \hat{T} \\
2 \theta(1-\theta) \hat{T} \geq \psi+\theta(1-\theta) T
\end{array}\right.
$$

Both constraints are binding at the optimum:

$$
T=\frac{1+2 \theta(1-\theta)}{\theta(1-\theta)(2 \theta-1)} \psi \quad \text { and } \quad U^{1,2}=\hat{T}=\frac{(2-\theta)}{(1-\theta)(2 \theta-1)} \psi .
$$

Finally, all other constraints are satisfied by the contract:

$$
\bar{t}=\frac{T}{2 \nu}, \quad \underline{\tau}=\frac{T}{2(1-\nu)}, \quad \tau_{1}=\frac{\hat{T}}{2 \nu} \quad \text { and } \quad \tau_{2}=\frac{\hat{T}}{2(1-\nu)} .
$$

Proof of Lemma 7: Consider an incentive scheme such that the project is undertaken with probability $\varepsilon\left(\hat{\sigma}_{1}, \hat{\sigma}_{2}\right)$ after reports $\left(\hat{\sigma}_{1}, \hat{\sigma}_{2}\right)$, and such that expert $i$ 's transfer $t_{i}\left(\hat{\sigma}_{i}, \hat{\sigma}_{j}, X\right)$ is contingent on the reports and the project's outcome. For brevity, we assume but do not prove that 
in the optimal contract, $t\left(\hat{\sigma}_{i}, \hat{\sigma}_{j}, \varnothing\right)=0$ and $t(\bar{\sigma}, \hat{\sigma}, \underline{S})=t(\underline{\sigma}, \hat{\sigma}, \bar{S})=0$. Denoting $\bar{t} \equiv t(\bar{\sigma}, \bar{\sigma}, \bar{S})$, $\tau_{G}=\varepsilon \times t(\bar{\sigma}, \underline{\sigma}, \bar{S}), \tau_{B}=\varepsilon \times t(\underline{\sigma}, \bar{\sigma}, \underline{S})$, and $\underline{\tau}=\varepsilon \times t(\underline{\sigma}, \underline{\sigma}, \underline{S})$, and $\bar{T} \equiv \nu\left(\theta \bar{t}+(1-\theta) \tau_{G}\right)$ and $\underline{T} \equiv(1-\nu)\left(\theta \underline{\tau}+(1-\theta) \tau_{B}\right)$, the principal's programme is:

$$
\min _{\bar{T} \geq 0, \underline{T} \geq 0} \theta(\bar{T}+\underline{T})
$$

subject to the following adverse selection incentive constraints:

$$
\theta \bar{T} \geq(1-\theta) \underline{T} \quad \text { and } \quad \theta \underline{T} \geq(1-\theta) \bar{T},
$$

which are implied by the moral hazard incentive constraints:

$$
\theta(\bar{T}+\underline{T})-\psi \geq \bar{T} \quad \text { and } \quad \theta(\bar{T}+\underline{T})-\psi \geq \underline{T} .
$$

Hence both constraints in (93) are binding at the optimum so that $\bar{T}=\underline{T}=\frac{\psi}{2 \theta-1}$, each expert's rent equals $\bar{T}$, and any contract with non negative transfers satisfying the following equalities is optimal:

$$
\nu\left(\theta \bar{t}+(1-\theta) \tau_{G}\right)=(1-\nu)\left(\theta \underline{\tau}+(1-\theta) \tau_{B}\right)=\frac{\psi}{2 \theta-1} .
$$

In particular, the optimal contract of the one-expert/one-signal case, $\bar{t}=\tau_{G}=\frac{\psi}{\nu(2 \theta-1)}$ and $\underline{\tau}=\tau_{B}=\frac{\psi}{(1-\nu)(2 \theta-1)}$, satisfies (94) and is robust to both ex ante and ex post horizontal collusion as it involves bilateral principal-expert contracts (i.e., $\bar{t}=\tau_{G}$ and $\underline{t}=\tau_{B}$ ). 